\title{
PATH-DEPENDENT CONVEX CONSERVATION LAWS
}

\author{
H. HOEL, K. H. KARLSEN, N. H. RISEBRO, AND E. B. STORRØSTEN
}

\begin{abstract}
For scalar conservation laws driven by a rough path $z(t)$, in the sense of Lions, Perthame and Souganidis in 34, we show that it is possible to replace $z(t)$ by a piecewise linear path, and still obtain the same solution at a given time, under the assumption of a convex flux function in one spatial dimension. This result is connected to the spatial regularity of solutions. We show that solutions are spatially Lipschitz continuous for a given set of times, depending on the path and the initial data. Fine properties of the map $z \mapsto$ $u(\tau)$, for a fixed time $\tau$, are studied. We provide a detailed description of the properties of the rough path $z(t)$ that influences the solution. This description is extracted by a "factorization" of the solution operator (at time $\tau$ ). In a companion paper [26], we make use of the observations herein to construct computationally efficient numerical methods.
\end{abstract}

\section{INTRODUCTION}

We are interested in scalar conservation laws of the form

$$
\left\{\begin{aligned}
\partial_{t} u+\partial_{x} f(u) \dot{z} & =0 & & \text { on }(0, T) \times \mathbb{R}, \\
u(0, x) & =u_{0}(x) & & \text { for } x \in \mathbb{R},
\end{aligned}\right.
$$

where $0<T<\infty$ is some fixed final time. The (rough) path $z:(0, \infty) \rightarrow \mathbb{R}$, the initial value $u_{0}: \mathbb{R} \rightarrow \mathbb{R}$, and the flux $f: \mathbb{R} \rightarrow \mathbb{R}$ are given functions, whereas $u$ is the unknown function that is sought. The time derivative of $z(t)$ is denoted by $\dot{z}$. Regarding the flux, the standing assumption is

$$
f \in C^{2} \text { and } f \text { is strictly convex. }
$$

Bear in mind that (1.1) reduces to a standard conservation law in the event $z(t)=t$. It is well known that such equations are well posed within the framework of Kružkov entropy solutions [12] or, equivalently, kinetic solutions [39]. More precisely, assuming for example $u_{0} \in\left(L^{\infty} \cap L^{1}\right)(\mathbb{R})$, there exists a unique function $u \in C\left([0, T] ; L^{1}(\mathbb{R})\right)$ satisfying $u(0, x)=u_{0}(x)$ and

$$
\partial_{t} S(u)+\partial_{x} Q(u) \dot{z} \leq 0 \quad \text { in the sense of distributions, }
$$

for all convex entropy, entropy-flux pairs $(S, Q)$, i.e., $S \in C^{2}$ convex and $Q^{\prime}=S^{\prime} f^{\prime}$.

If $z(t)$ is a Brownian path (i.e., a realization of a Brownian motion), then $z(t)$ is merely Hölder continuous (infinite variation) and the conservation law (1.1) is

Date: December 27, 2017.

2010 Mathematics Subject Classification. Primary: 35L65; Secondary: 60H15.

Key words and phrases. Hyperbolic conservation law, rough time-dependent flux, stochastic PDE, pathwise entropy solution, regularity.

This work was supported by the Research Council of Norway (project 250674/F20). 
no longer well defined; in this case one could replace 1.1 by the stochastic partial differential equations (SPDE)

$$
\partial_{t} u+\partial_{x} f(u) \circ d z=0
$$

where $\circ$ denotes the Stratonovich differential. Aiming for a different approach, Lions, Perthame, and Souganidis 34 recently introduced a pathwise notion of entropy/kinetic solution to [1.1), defined for any $z \in C([0, T])$, which is consistent with the notion of Kružkov entropy solution for regular paths $z(t)$.

According to [34], $u \in L^{\infty}\left([0, T] ; L^{1}(\mathbb{R})\right) \cap L^{\infty}(\mathbb{R} \times[0, T])$ is called a pathwise entropy/kinetic solution to (1.1) provided there is a non-negative, bounded measure $m$ on $\mathbb{R} \times \mathbb{R} \times[0, T]$ such that for all $\rho \in C_{c}^{\infty}(\mathbb{R}), \chi(\xi, u):=\mathbb{1}_{0<\xi<u}-\mathbb{1}_{u<\xi<0}$ satisfies

$$
\partial_{t} \int_{\mathbb{R}} \chi(\xi, u(t, x)) \rho\left(x-f^{\prime}(\xi) z(t)\right) d x=\int_{\mathbb{R}} \rho\left(x-f^{\prime}(\xi) z(t)\right) \partial_{\xi} m(t, x, \xi) d x
$$

in the weak sense on $\mathbb{R} \times[0, T]$. Informally, the motivation behind the notion of pathwise solutions (1.4) comes from writing the usual kinetic formulation of (1.1),

$$
d \chi+f^{\prime}(\xi) \cdot \nabla \chi \dot{z}=\partial_{\xi} m,
$$

for a bounded measure $m(t, x, \xi) \geq 0$ and a function $u(t, x)$ (entropy solution) such that $\chi=\chi(t, x, \xi):=\chi(\xi, u(t, x))$. Next, one uses the "method of characteristics" to remove the rough path. The result is that the function

$$
v=v(t, x, \xi):=\chi\left(t, x+f^{\prime}(\xi) z(t), \xi\right)
$$

satisfies the following kinetic equation without the rough drift term:

$$
\begin{aligned}
\partial_{t} v= & \left(\partial_{\xi} m\right)\left(t, x+f^{\prime}(\xi) z(t), \xi\right) \\
= & \partial_{\xi}\left(m\left(t, x+f^{\prime}(\xi) z(t), \xi\right)\right) \\
& \quad-f^{\prime \prime}(\xi) z(t)\left(\partial_{x} m\right)\left(t, x+f^{\prime}(\xi) z(t), \xi\right) .
\end{aligned}
$$

The defining equation (1.4) constitutes a weak formulation of (1.5). Various results concerning existence, uniqueness, and stability of pathwise solutions are found in the works [35, 34, 36]. The theory of pathwise solutions has been further developed in 24, 25, see also [28] and [4, 16].

It is proved in 34 that the pathwise solution is stable with respect to uniform convergence of the path. More precisely, assuming $u_{0} \in B V(\mathbb{R})\left(u_{0}\right.$ is of bounded variation), we have the following result [34, Theorem 3.2]: Let $u^{i}$ be the pathwise entropy/kinetic solution of 1.1 with path $z^{i}$ and initial condition $u_{0}^{i}$, for $i=1,2$. Then there is a constant $C>0$ such that for $t \in[0, T]$,

$$
\begin{aligned}
& \left\|u^{1}(t)-u^{2}(t)\right\|_{L^{1}(\mathbb{R})} \leq\left\|u_{0}^{1}-u_{0}^{2}\right\|_{L^{1}(\mathbb{R})} \\
& +C\left[\left\|f^{\prime}\right\|_{\infty}\left(\left\|u_{0}^{1}\right\|_{B V(\mathbb{R})}+\left\|u_{0}^{2}\right\|_{B V(\mathbb{R})}\right)\left|z^{1}(t)-z^{2}(t)\right|\right. \\
& \left.\quad+\sqrt{\sup _{s \in(0, t)}\left|z^{1}(s)-z^{2}(s)\right|\left\|f^{\prime \prime}\right\|_{\infty}\left(\left\|u_{0}^{1}\right\|_{L^{2}(\mathbb{R})}^{2}+\left\|u_{0}^{2}\right\|_{L^{2}(\mathbb{R})}^{2}\right)}\right],
\end{aligned}
$$

Consequently, given a sequence of regular (say, Lipschitz) paths $\left\{z^{n}\right\}_{n \geq 0}$ converging uniformly to $z$ as $n \rightarrow \infty$, the corresponding Kružkov entropy solutions $\left\{u^{n}\right\}_{n \geq 0}$ of (1.1) converges to the entropy/kinetic pathwise solution $u$ in $C\left([0, T] ; L^{1}(\mathbb{R})\right)$ as $n \rightarrow \infty$. As such, the interpretation of (1.1) in terms of (1.4) is associated with 
the Stratonovich interpretation of (1.1). In view of the consistency between (1.2) and 1.4, in what follows we will refer to Kružkov entropy solutions and pathwise entropy/kinetic solutions both simply as entropy solutions.

In this paper, we approximate the entropy solution $u$ of $(1.1)$ by a sequence $\left\{u^{n}\right\}_{n \geq 0}$ of solutions utilizing piecewise linear approximations $\left\{z^{n}\right\}_{n \geq 0}$ of the rough path $z \in C([0, T])$. The "continuous dependence on the data" estimate (1.6) ensures that this approximation converges to the correct solution of (1.1). A motivation for exploring such approximations is their relevance to numerical methods. The computational difficulties associated with solving (1.1) numerically stem from the infinite variation of the rough path $z(t)$. This forces the time step to be very small due to the well-known CFL stability condition, linking the temporal and spatial discretization parameters. Our main result, valid for convex flux functions $f$, states that it is possible to replace the rough path by a piecewise linear path of finite variation, and still obtain the same solution at a fixed time. In [26] we make use of this result to construct computationally efficient finite volume methods.

Let us discuss in more detail our results relating to path-dependence of entropy solutions. Suppose the path $z(t)$ is piecewise linear and continuous, and let $u$ be the corresponding entropy solution to (1.1). Fix a time $\tau \in[0, T]$. We seek the "simplest" path $\tilde{z}$ such that the corresponding solution $\tilde{u}$ satisfies $\tilde{u}(\tau, \cdot)=u(\tau, \cdot)$. To motivate, fix a time interval $\left[t_{1}, t_{2}\right]$ with $0 \leq t_{1}<t_{2} \leq \tau$, and suppose for simplicity that $z(t) \geq z\left(t_{1}\right)$ on $\left[t_{1}, t_{2}\right]$. Let $v$ be the entropy solution to

$$
\partial_{t} v+\partial_{x} f(v)=0, \quad v\left(z\left(t_{1}\right), x\right)=u\left(t_{1}, x\right) .
$$

Then $u(t, x)=v(z(t), x)$ on $\left[t_{1}, t_{2}\right]$ in either of the two following cases:

(i) $z(t)$ is monotone on $\left[t_{1}, t_{2}\right]$.

(ii) $v$ is a classical solution (no shocks) on $\left\{z(t): t \in\left[t_{1}, t_{2}\right]\right\}$.

Consequently, we may replace parts of the path $z$ satisfying either (i) or (ii) by straight line segments, i.e., $z$ by the path

$$
\tilde{z}(t):=\left\{\begin{array}{l}
z\left(t_{1}\right)+\left(t-t_{1}\right) \frac{z\left(t_{2}\right)-z\left(t_{1}\right)}{t_{2}-t_{1}} \text { for } t \in\left[t_{1}, t_{2}\right], \\
z(t) \text { else. }
\end{array}\right.
$$

As the solution $\tilde{u}$ (corresponding to $\tilde{z}$ ) satisfies $\tilde{u}\left(t_{2}\right)=v\left(\tilde{z}\left(t_{2}\right)\right)=v\left(z\left(t_{2}\right)\right)=u\left(t_{2}\right)$ it follows that $u(\tau)=\tilde{u}(\tau)$. In view of this, we can "simplify" the path $z(t)$ by replacing the parts where it satisfies either $(i)$ or $(i i)$ by straight line segments. It is easy to determine which parts satisfies (i), i.e., where the path is monotone. Considering (ii), we need to determine the parts of the path $z$ on which $u$ (and $v$ ) is a classical solution (without shocks). To this end, let us recall the so-called Oleĭnik estimate for strictly convex fluxes $f$ (and $\dot{z}=1$ ) [12]:

$$
\frac{f^{\prime}(u(t, y))-f^{\prime}(u(t, x))}{y-x} \leq \frac{1}{t}, \quad y>x, t>0 .
$$

For example with $f=u^{2} / 2$, the only admissible shocks are those for which the left value is larger than the right. Similarly, with $f=u^{2} / 2$ and $\dot{z}=-1$, only upward jumping shocks are admissible. When $z^{\prime}(t)$ oscillates (i.e., takes on positive and negative values, say \pm 1 ), one observes that shocks in $u$ can only exist when the path $z$ takes on values not already assumed at some earlier point in time. Consequently, starting from a piecewise linear path $z$, it is possible to "inductively" construct a new path $\tilde{z}(t)$, with smaller total variation, by replacing appropriate parts of 
$z(t)$ by linear segments. This inductive procedure, which we describe in detail in an upcoming section, gives rise to a "minimal" path associated with the final time $\tau$ and the initial data $u_{0}$. We refer to the resulting path as the oscillating running min/max path and label it $\operatorname{Orm}_{\tau, u_{0}}(z)$ (See Definition 2.2 below). Setting $\tilde{z}=\operatorname{Orm}_{\tau, u_{0}}(z)$, we have $\tilde{u}(\tau)=u(\tau)$. Note that the application of the Oleunik estimate depended on $\dot{z}$ being piecewise constant. However, for sufficiently smooth $u_{0}$, it turns out that that $z \mapsto \operatorname{Orm}_{\tau, u_{0}}(z)$ is well-defined for any continuous path $z$. Indeed, for a general path $z$, we proceed by suitable piecewise linear approximation.

This approach may be viewed as a factorization of the solution operator. To see how this is related to the construction of the map $z \mapsto \operatorname{Orm}_{\tau, u_{0}}(z)$, we identify two paths $z$ and $\tilde{z}$ as long as $u(\tau)=\tilde{u}(\tau)$, where $z \mapsto u(\tau)$ and $\tilde{z} \mapsto \tilde{u}(\tau)$. This naturally leads to a factorization of the solution operator (one for each fixed time $\tau$ ) as a composition of a quotient map and an injective map, see Figure 2. Up to precomposition by a nondecreasing function, the quotient map may be identified with the map $z \mapsto \operatorname{Orm}_{\tau, u_{0}}(z)$. The injective map is associated with the solution operator restricted to piecewise linear paths.

Another question raised in this work is related to the optimal choice of paths relative to the continuous dependence estimate 1.6 . To be more precise, in view of the above discussion, there exists for each path $z$ a multitude of paths $\tilde{z}$ such that, for a given time $\tau \in[0, T]$ and initial condition $u_{0}$, the corresponding solutions $u$ and $\tilde{u}$ to 1.1 satisfy $u(\tau)=\tilde{u}(\tau)$. In order to improve (1.6) one may search for paths $\tilde{z}_{1}, \tilde{z}_{2}$ satisfying $u^{1}(\tau)=\tilde{u}^{1}(\tau), u^{2}(\tau)=\tilde{u}^{2}(\tau)$ such that

$$
\sup _{0 \leq t \leq \tau}\left\{\left|\tilde{z}_{1}(t)-\tilde{z}_{2}(t)\right|\right\} \text { is as small as possible. }
$$

In Theorem 2.10 below, it is shown that this minimization problem may be bounded in terms of a second minimization problem solvable by dynamic programming.

Before ending this introduction, we mention that recently many researchers studied the effect of adding randomness to conservation laws and other related nonlinear partial differential equations. This includes stochastic transport equations, which bears some resemblance to 1.3 ,

$$
d u+b(x) \cdot \nabla u d t+\nabla u \circ d W(t)=0
$$

where $b(x)$ is a low-regularity velocity field and the "transportation noise" is driven by a Wiener process $W(t)$. For some representative results, see e.g. [3, 21, 37, 38. In a different direction, many mathematical papers $[8,0,11,13,14,15,19,27,32$, 30, 20, 41, 40 have studied the effect of Itô stochastic forcing on conservation laws,

$$
d u+\nabla \cdot f(u) d t=\sigma(u) d W(t)
$$

where $f, \sigma$ are nonlinear functions and $W(t)$ is a (finite or infinite dimensional) Wiener process. Numerical methods are looked at in [2, 7, 6, 5, 29, 31, 17, 18, 33.

The remaining part of this paper is organized as follows: In Section 2, the main results of the paper are presented, without proofs, along with the notation necessary to make the statements precise. In Section 3 proofs of the given results are presented. 


\section{MAin Results}

To state the main results precisely, we introduce some notation and definitions. The regularity of $u_{0}$ is quantified by two numbers $0 \leq M_{+}, M_{-} \leq \infty$ satisfying

$$
-M_{-} \leq \frac{f^{\prime}\left(u_{0}(y)\right)-f^{\prime}\left(u_{0}(x)\right)}{y-x} \leq M_{+}, \quad x, y \in \mathbb{R}, x<y .
$$

Denote by $C_{0}([0, T])=\{z \in C([0, T]): z(0)=0\}$ the space of continuous paths starting at the origin. For a given path $z \in C_{0}([0, T])$, we introduce the "truncated" running $\min / \max$ functions, which are defined by

$$
\rho_{z}^{+}(t):=\max \left\{\frac{1}{M_{-}}, \max _{0 \leq s \leq t}\{z(s)\}\right\} \text { and } \rho_{z}^{-}(t):=\min \left\{-\frac{1}{M_{+}}, \min _{0 \leq s \leq t}\{z(s)\}\right\},
$$

cf. Figure 2 Here we use the convention that $0^{-1}=\infty$. Consequently, if $M_{-}=0$, then $\rho_{z}^{+}(t)=\infty$ for all $t \in[0, \tau]$. A path $z \in C_{0}([0, T])$ is called piecewise linear if there is a finite sequence $\left\{t_{n}\right\}_{n=0}^{N}$ with $0=t_{0}<t_{1}<\cdots<t_{N}=T$, such that

$$
z(t)=z\left(t_{n}\right)+\left(t-t_{n}\right) \frac{z\left(t_{n+1}\right)-z\left(t_{n}\right)}{t_{n+1}-t_{n}} \quad \text { for } t \in\left[t_{n}, t_{n+1}\right] .
$$

Furthermore, we define the sets where $\rho_{z}^{ \pm}$is strictly increasing/decreasing by

$$
\begin{aligned}
& \mathscr{B}_{z}^{+}:=\left\{t \in[0, T]: \inf \left\{s \in[0, T]: \rho_{z}^{+}(s) \geq \rho_{z}^{+}(t)\right\}=t\right\}, \\
& \mathscr{B}_{z}^{-}:=\left\{t \in[0, T]: \inf \left\{s \in[0, T]: \rho_{z}^{-}(s) \leq \rho_{z}^{-}(t)\right\}=t\right\} .
\end{aligned}
$$

The next lemma summarizes the essential properties of these sets.

Lemma 2.1. Let $\mathscr{B}_{z}^{ \pm}$be defined by 2.3. Then

(i) $\mathscr{B}_{z}^{ \pm} \subset\left\{t \in[0, T]: z(t)=\rho_{z}^{ \pm}(t)\right\} \cup\{0\}$. Furthermore $\mathscr{B}_{z}^{+} \cap \mathscr{B}_{z}^{-}=\{0\}$.

(ii) $\mathscr{B}_{z}^{ \pm}$are closed with respect to increasing sequences, i.e., if $\left\{t_{n}\right\}_{n \geq 0} \subset \mathscr{B}_{z}^{ \pm}$ satsifies $t_{n} \uparrow t$ for some $t \geq 0$, then $t \in \mathscr{B}_{z}^{ \pm}$. Hence

$$
\sup \left\{\mathscr{B}_{z}^{ \pm} \cap[0, \tau]\right\} \in \mathscr{B}_{z}^{ \pm} \text {for any } \tau \in[0, T] .
$$

(iii) Let $0 \leq t_{1}<t_{2} \leq T$. Suppose $\left(t_{1}, t_{2}\right] \cap \mathscr{B}_{z}^{ \pm}=\emptyset$. Then $\rho_{z}^{ \pm}$is constant on $\left[t_{1}, t_{2}\right]$ respectively.

(iv) Suppose $\rho_{z}^{ \pm}$is left differentiable on $(0, T]$. Then

$$
\begin{aligned}
\mathscr{B}_{z}^{+} & =\mathrm{cl}_{-}\left(\left\{t \in(0, T]: \partial_{-} \rho_{z}^{+}(t)>0\right\}\right) \cup\{0\}, \\
\mathscr{B}_{z}^{-} & =\mathrm{cl}_{-}\left(\left\{t \in(0, T]: \partial_{-} \rho_{z}^{-}(t)<0\right\}\right) \cup\{0\},
\end{aligned}
$$

where $\partial_{-}$denotes the left derivative and $\mathrm{cl}_{-}$denotes the closure with respect to increasing sequences. Consequently, for piecewise linear $z$ there exists $0 \leq N^{ \pm}<\infty$ and $0 \leq s_{1}^{ \pm}<t_{1}^{ \pm}<\cdots<s_{N^{ \pm}}^{ \pm}<t_{N^{ \pm}}^{ \pm} \leq T$ such that

$$
\mathscr{B}_{z}^{ \pm} \backslash\{0\}=\bigcup_{n=1}^{N^{ \pm}}\left(s_{n}^{ \pm}, t_{n}^{ \pm}\right],
$$

where we use the convention that the union is empty if $N^{ \pm}=0$.

We may now give the precise definition of the Oscillating Running Min/Max. 
Definition 2.2 (Oscillating Running Min/Max). Given a path $z \in C_{0}([0, T]$ ), define the sequence $\left\{\tau_{n}\right\}_{n \geq 0}$ inductively by

$$
\begin{aligned}
\tau_{0} & =\tau, \\
\tau_{n+1} & = \begin{cases}\max \left\{\mathscr{B}_{z}^{+} \cap\left[0, \tau_{n}\right]\right\} & \text { if } \tau_{n} \in \mathscr{B}_{z}^{-}, \\
\max \left\{\mathscr{B}_{z}^{-} \cap\left[0, \tau_{n}\right]\right\} & \text { if } \tau_{n} \in \mathscr{B}_{z}^{+}, \\
\max \left\{\left(\mathscr{B}_{z}^{+} \cup \mathscr{B}_{z}^{-}\right) \cap\left[0, \tau_{n}\right]\right\} & \text { if } \tau_{n} \notin \mathscr{B}_{z}^{+} \cup \mathscr{B}_{z}^{-},\end{cases}
\end{aligned}
$$

for $n=0,1,2, \ldots$ If there exists an integer $0 \leq N<\infty$ such that $\tau_{N}=0$, then we define $\operatorname{Orm}_{\tau, M_{ \pm}}(z)$ (Oscillating Running Min/Max) as the piecewise linear interpolation of $\left\{\left(\tau_{n}, z\left(\tau_{n}\right)\right)\right\}_{n=0}^{N}$.

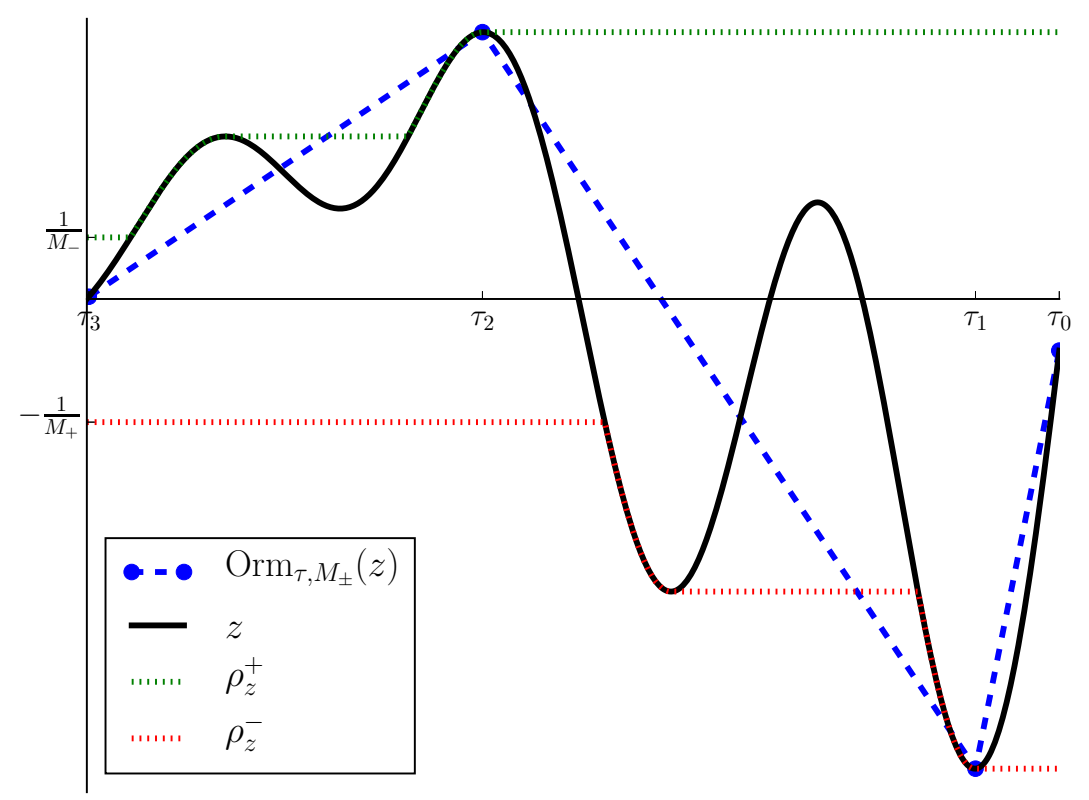

Figure 1. Illustration of $\rho_{z}^{+}, \rho_{z}^{-}$and $\operatorname{Orm}_{\tau, M_{ \pm}}(z)$ for a given path $z$.

Even though the Oscillating Running Min/Max only depends on $z, \tau, M_{-}, M_{+}$, we are often interested in a specific initial condition $u_{0}$ satisfying (2.1) for some given numbers $0 \leq M_{-}, M_{+} \leq \infty$. In such situations we often write $\operatorname{Orm}_{\tau, u_{0}}(z)$ instead of $\operatorname{Orm}_{\tau, M_{ \pm}}(z)$. Let us mention that $\operatorname{Orm}_{\tau, M_{ \pm}}(z)$ is well defined for any path $z \in C_{0}([0, T])$, given that $0 \leq \min \left\{M_{+}, M_{-}\right\}<\infty$, see Lemma 3.9. In view of the above discussion, there emerges a natural equivalence relation on the set of paths. For convenience, the relation is here defined on an arbitrary interval.

Definition 2.3 (Equivalence of paths). Fix a time interval $\left[t_{1}, t_{2}\right] \subset[0, \infty)$ and two paths $z_{1}, z_{2} \in C\left(\left[t_{1}, t_{2}\right]\right)$. Let $u^{i}$ be the entropy solution to

$$
\partial_{t} u^{i}+\partial_{x} f\left(u^{i}\right) \dot{z}_{i}=0, \quad u^{i}\left(t_{1}\right)=u_{0} \in\left(L^{1} \cap L^{\infty}\right)(\mathbb{R}),
$$

on $\left[t_{1}, t_{2}\right]$ for $i=1,2$. If $u^{1}\left(t_{2}\right)=u^{2}\left(t_{2}\right)$, we say that $z_{1}$ is equivalent to $z_{2}$, written $z_{1} \sim z_{2}$, on $\left[t_{1}, t_{2}\right]$ with initial condition $u_{0}$. 
We are now ready to state the result alluded to above.

Theorem 2.4. Let $f$ satisfy $\left(\overrightarrow{\mathcal{A}_{f}}\right), z \in C_{0}([0, \tau])$. If $\mathrm{Orm}_{\tau, M_{ \pm}}(z)$ is well-defined, then $z \sim \operatorname{Orm}_{\tau, M_{ \pm}}(z)$ on $[0, \tau]$ for any $u_{0} \in\left(L^{1} \cap L^{\infty}\right)(\mathbb{R})$ satisfying $(2.1)$.

As mentioned above, for piecewise linear paths, the Olel̆nik estimate implies that the solution $u(t)$ is (spatially Lipschitz) continuous for $t$ in certain regions of the path. In the following theorem this result is extended, via Theorem 2.4 to the case of a general path $z \in C_{0}([0, T])$.

Theorem 2.5. Assume $f$ satisfies $\left(\sqrt[\mathcal{A}_{f}]{ }\right), z \in C_{0}([0, T])$ and $u_{0} \in\left(L^{1} \cap L^{\infty}\right)(\mathbb{R})$. Let $u$ be the entropy solution to (1.1). Then, for any $t \in[0, T]$,

$$
-\frac{1}{\rho_{z}^{+}(t)-z(t)} \leq \frac{f^{\prime}(u(t, y \pm))-f^{\prime}(u(t, x \pm))}{y-x} \leq \frac{1}{z(t)-\rho_{z}^{-}(t)},
$$

for all $-\infty<x<y<\infty$. Here, $u(t, x \pm)$ denotes respectively the right and left limits. We apply the convention $(\infty)^{-1}=0$ and $(0)^{-1}=\infty$.

Remark 2.6. Apriori, the left/right limits should be interpreted as essential limits and the statement should be restricted to points $-\infty<x<y<\infty$ such that these limits exists. However, whenever the lower or upper bound is finite, it implies that $u(t, \cdot)$ belongs to $B V_{\text {loc }}(\mathbb{R})$ and the left/right limits exist in the classical sense.

Remark 2.7. In [22] the authors investigate regularity properties of solutions to the equation

$$
d v+\frac{1}{2}|D v|^{2} \circ d z=F\left(x, v, D v, D^{2} v\right) d t
$$

where $z$ is a continuous path, and $F$ is a nonlinear function meeting the standard assumptions from the theory of viscosity solutions of fully nonlinear degenerate parabolic PDEs. An $L^{\infty}$-bound on the second derivative $D^{2} v$ is established in [22]. In the special case

$$
d u+\partial_{x}\left(\frac{u^{2}}{2}\right) \circ d z=0, \quad u=\partial_{x} v,
$$

this estimate reduces to the Lipschitz $\left(W^{1, \infty}\right)$ bound

$$
\underset{x \neq y}{\operatorname{ess} \sup _{x}}\left|\frac{u(x)-u(y)}{x-y}\right| \leq \frac{1}{\max _{s \in[0, t]} z(s)-z(t)} \vee \frac{1}{z(t)-\min _{s \in[0, t]} z(s)} .
$$

This estimate is similar to the one provided by Theorem 2.5 which in the special case $f(u)=u^{2} / 2$ can be recast as

$$
-\frac{1}{\rho_{z}^{+}(t)-z(t)} \leq \frac{u(t, x)-u(t, y)}{x-y} \leq \frac{1}{z(t)-\rho_{z}^{-}(t)}, \quad \text { for a.e. } x, y \in \mathbb{R} .
$$

Although the results are similar, both relying on the strict convexity of the flux but with the one in $\left[22\right.$ restricted to $f=u^{2} / 2$, the proofs are different. We work at the level of conservation laws and use the method of generalized characteristics. The argument in 22 relies on semiconvexity preservation properties of Hamilton-Jacobi equations.

\footnotetext{
${ }^{1}$ We became aware of the work 22 in the final stage of writing this paper.
} 
In view of Theorem 2.4 the equivalence class of a given path is nontrivial. The following result yields a condition sufficient for two paths to be equivalent. Let

$$
\mathscr{A}_{\tau}:=\{\alpha:[0, \tau] \rightarrow[0, \tau]: \alpha \text { is nondecreasing and surjective }\}
$$

Theorem 2.8. Let $f$ satisfy $\left(\mathcal{A}_{f}\right)$. Fix $z_{1}, z_{2} \in C_{0}([0, \tau])$ such that $z_{1}(\tau)=z_{2}(\tau)$. Suppose there exist paths $\alpha_{i} \in \mathscr{A}_{\tau}, i=1,2$, such that

$$
\rho_{z_{1}}^{+} \circ \alpha_{1}=\rho_{z_{2}}^{+} \circ \alpha_{2} \quad \text { and } \quad \rho_{z_{1}}^{-} \circ \alpha_{1}=\rho_{z_{2}}^{-} \circ \alpha_{2}
$$

Then $z_{1} \sim z_{2}$ on $[0, \tau]$ for all $u_{0} \in\left(L^{\infty} \cap L^{1}\right)(\mathbb{R})$ satisfying 2.1 .

Remark 2.9. We note that the existence of $\alpha_{1}, \alpha_{2}$ is closely related to the problem of optimal transport (on $\mathbb{R}$ ) 42. Here we have two (continuous) transference plans, represented by $\alpha_{1}, \alpha_{2}$, which should satisfy two transportation problems. Recall that $\rho_{z_{i}}^{+}$is constant whenever $\rho_{z_{i}}^{-}$is decreasing, while $\rho_{z_{i}}^{-}$is constant as long as $\rho_{z_{i}}^{+}$ is increasing, $i=1,2$.

It seems likely that the condition 2.5 is also necessary, at least on a more restricted space of paths, cf. Lemma 3.8. For $z_{1}, z_{2}$ as in Theorem 2.8, we write $z_{1} \sim_{\circ} z_{2}$ if there exist $\alpha_{1}, \alpha_{2} \in \mathscr{A}_{\tau}$, such that $\rho_{z_{1}}^{ \pm} \circ \alpha_{1}=\rho_{z_{2}}^{ \pm} \circ \alpha_{2}$. It is obvious that the relation $\sim_{0}$ is both reflexive and symmetric, i.e., that $z_{1} \sim_{\circ} z_{1}$ and $z_{1} \sim_{0} z_{2}$ implies $z_{2} \sim_{0} z_{1}$. To check that it is transitive, fix $z_{1}, z_{2}, z_{3} \in C_{0}([0, \tau])$ such that $z_{1} \sim_{0} z_{2}$ and $z_{2} \sim_{0} z_{3}$, i.e., suppose there exist nondecreasing surjective maps $\alpha_{i}, \beta_{i}, i=1,2$, such that

$$
\rho_{z_{1}}^{ \pm} \circ \alpha_{1}=\rho_{z_{2}}^{ \pm} \circ \alpha_{2} \quad \text { and } \quad \rho_{z_{2}}^{ \pm} \circ \beta_{1}=\rho_{z_{3}}^{ \pm} \circ \beta_{2}
$$

Then there exist, at last in the piecewise linear setting, nondecreasing surjective maps $\zeta_{1}, \zeta_{2}$ such that $\alpha_{2} \circ \zeta_{1}=\beta_{1} \circ \zeta_{2}$, cf. Lemma 3.8. Hence,

$$
\rho_{z_{1}}^{ \pm} \circ\left(\alpha_{1} \circ \zeta_{1}\right)=\rho_{z_{2}}^{ \pm} \circ \alpha_{2} \circ \zeta_{1}=\rho_{z_{2}}^{ \pm} \circ \beta_{1} \circ \zeta_{2}=\rho_{z_{3}}^{ \pm} \circ\left(\beta_{2} \circ \zeta_{2}\right),
$$

and so $z_{1} \sim_{\circ} z_{3}$.

For a path $z \in C_{0}([0, \tau])$, we denote its equivalence class with respect to $\tau$ and $M_{ \pm}$by $[z]_{\tau, M_{ \pm}}$, that is,

$$
\left.[z]_{\tau, M_{ \pm}}=\left\{\tilde{z} \in C_{0}([0, \tau]): \tilde{z} \sim z \text { on }[0, \tau] \text { for any } u_{0} \text { satisfying } 2.1\right]\right\}
$$

Fix $0 \leq M_{+}^{i}, M_{-}^{i} \leq \infty, i=1,2$. Regarding the continuous dependence estimate (1.6), one may exchange the uniform distance between two paths by the distance between two equivalence classes:

$$
\begin{aligned}
\|\left[z_{1}\right]_{\tau, M_{ \pm}^{1}} & -\left[z_{2}\right]_{\tau, M_{ \pm}^{2}} \|_{\infty} \\
& :=\inf \left\{\left\|\zeta_{1}-\zeta_{2}\right\|_{\infty}: \zeta_{1}, \zeta_{2} \in C_{0}([0, \tau]), \zeta_{1} \stackrel{\tau, M_{ \pm}^{1}}{\sim} z_{1}, \zeta_{2} \stackrel{\tau, M_{ \pm}^{2}}{\sim} z_{2}\right\}
\end{aligned}
$$


where $\zeta \stackrel{\tau, M_{ \pm}^{i}}{\sim} z$ is shorthand notation for $\zeta \sim z$ on $[0, \tau]$ for any initial condition $u_{0}$ satisfying (2.1) with $M_{ \pm}=M_{ \pm}^{j}$. Therefore, (1.6) may be replaced by

$$
\begin{aligned}
& \left\|u^{1}(\tau)-u^{2}(\tau)\right\|_{L^{1}(\mathbb{R})} \leq\left\|u_{0}^{1}-u_{0}^{2}\right\|_{L^{1}(\mathbb{R})} \\
& +C\left[\left\|f^{\prime}\right\|_{\infty}\left(\left\|u_{0}^{1}\right\|_{B V(\mathbb{R})}+\left\|u_{0}^{2}\right\|_{B V(\mathbb{R})}\right)\left|z^{1}(\tau)-z^{2}(\tau)\right|\right. \\
& \left.\quad+\sqrt{\left\|\left[z^{1}\right]_{\tau, M_{ \pm}^{1}}-\left[z^{2}\right]_{\tau, M_{ \pm}^{2}}\right\|_{\infty}\left\|f^{\prime \prime}\right\|_{\infty}\left(\left\|u_{0}^{1}\right\|_{L^{2}(\mathbb{R})}^{2}+\left\|u_{0}^{2}\right\|_{L^{2}(\mathbb{R})}^{2}\right)}\right]
\end{aligned}
$$

for $u_{0}^{i}$ satisfying (2.1) with $M_{ \pm}=M_{ \pm}^{i}, i=1,2$. Assuming $M_{ \pm}=M_{ \pm}^{1}=M_{ \pm}^{2}$, in view of Theorem 2.8 one may hypothesize that the distance 2.6) can be estimated in terms of the minimization problem

$$
\begin{aligned}
& \left\|\left[z_{1}\right]_{\tau, M_{ \pm}}-\left[z_{2}\right]_{\tau, M_{ \pm}}\right\|_{\infty} \\
& \leq \inf _{\alpha_{1}, \alpha_{2} \in \mathscr{A}_{\tau}}\left\{\max _{ \pm}\left\{\left\|\rho_{z_{1}}^{ \pm} \circ \alpha_{1}-\rho_{z_{2}}^{ \pm} \circ \alpha_{2}\right\|_{\infty},\left|z_{1}(\tau)-z_{2}(\tau)\right|\right\}\right\} .
\end{aligned}
$$

Our next result shows that this is indeed the case. To make the statement precise, we need to introduce some notation. Suppose $z \in C_{0}([0, \tau])$ is such that $\operatorname{Orm}_{\tau, M_{ \pm}}(z)$ is well defined. Denote by $\mathcal{T}_{z}:=\left\{\tau_{n}\right\}_{n=0}^{N}$ the interpolation points associated with $\operatorname{Orm}_{\tau, M_{ \pm}}(z)$, cf. Definition 2.2, and set

$$
\mathcal{T}_{z}^{ \pm}:=\mathcal{T}_{z} \cap \mathscr{B}_{z}^{ \pm} \backslash\{0\} .
$$

According to Lemma 2.1 (i),

$$
\mathcal{T}_{z}^{ \pm}=\left\{\tau_{n}: 0 \leq n \leq N-1 \text { such that } z\left(\tau_{n}\right)=\rho_{z}^{ \pm}\left(\tau_{n}\right)\right\} .
$$

For any $\alpha \in \mathscr{A}_{\tau}, \mathcal{T}_{z \circ \alpha}^{ \pm}=\left\{\alpha^{-1}(t): t \in \mathcal{T}_{z}^{ \pm}\right\}$, where $\alpha^{-1}$ denotes the generalized inverse of $\alpha$, i.e.,

$$
\alpha^{-1}(t):=\inf \{s \in[0, \tau]: \alpha(s) \geq t\} .
$$

Theorem 2.10. Suppose $f$ satisfies $\left(\mathcal{A}_{f}\right)$. Let $0<\tau \leq T, z_{1}, z_{2} \in C_{0}([0, \tau])$ and $0 \leq M_{+}^{i}, M_{-}^{i}<\infty, i=1,2$. Let $\rho_{z_{i}}^{ \pm}$be defined with respect to $M_{ \pm}^{i}, i=1,2$, cf. 2.2. Then, for any $\alpha_{1}, \alpha_{2} \in \mathscr{A}_{\tau}$, define

$$
\begin{aligned}
& \Phi\left[z_{1}, z_{2}\right]\left(\alpha_{1}, \alpha_{2}\right) \\
& :=\max \left(\begin{array}{l}
\left\{\left|\rho_{z_{1}}^{+} \circ \alpha_{1}(t)-\rho_{z_{2}}^{+} \circ \alpha_{2}(t)\right|: t \in \mathcal{T}_{z_{1} \circ \alpha_{1}}^{+} \cup \mathcal{T}_{z_{2} \circ \alpha_{2}}^{+} \cap\left(\kappa^{+}, \tau\right]\right\} \\
\cup\left\{\left|\rho_{z_{1}}^{-} \circ \alpha_{1}(t)-\rho_{z_{2}}^{-} \circ \alpha_{2}(t)\right|: t \in \mathcal{T}_{z_{1} \circ \alpha_{1}}^{-} \cup \mathcal{T}_{z_{2} \circ \alpha_{2}}^{-} \cap\left(\kappa^{-}, \tau\right]\right\} \\
\cup\left\{\left|z_{1}(\tau)-z_{2}(\tau)\right|\right\}
\end{array}\right),
\end{aligned}
$$

where $\kappa^{ \pm}=\kappa_{z_{1}, z_{2}}^{ \pm}\left(\alpha_{1}, \alpha_{2}\right)$ are defined by

$$
\begin{aligned}
& \kappa^{+}=\inf \left\{t \in[0, \tau]: \max \left\{\rho_{z_{1}}^{+} \circ \alpha_{1}(t), \rho_{z_{2}}^{+} \circ \alpha_{2}(t)\right\}>\max \left\{\frac{1}{M_{-}^{1}}, \frac{1}{M_{-}^{2}}\right\}\right\}, \\
& \kappa^{-}=\inf \left\{t \in[0, \tau]: \min \left\{\rho_{z_{1}}^{-} \circ \alpha_{1}(t), \rho_{z_{2}}^{-} \circ \alpha_{2}(t)\right\}<\min \left\{-\frac{1}{M_{+}^{1}},-\frac{1}{M_{+}^{2}}\right\}\right\},
\end{aligned}
$$


with the convention that $\kappa^{ \pm}=\infty$ if the set is empty. Set $\iota(t)=t$. Then

$$
\begin{aligned}
\left\|\left[z_{1}\right]_{\tau, M_{ \pm}^{1}}-\left[z_{2}\right]_{\tau, M_{ \pm}^{2}}\right\|_{\infty} & =\inf \left\{\Phi\left[\tilde{z}_{1}, \tilde{z}_{2}\right](\iota, \iota): \tilde{z}_{1} \stackrel{\tau, M_{ \pm}^{1}}{\sim} z_{1}, \tilde{z}_{2} \stackrel{\tau, M_{ \pm}^{2}}{\sim} z_{2}\right\} \\
& \leq \inf \left\{\Phi\left[\tilde{z}_{1}, \tilde{z}_{2}\right](\iota, \iota): \tilde{z}_{1} \stackrel{\tau, M_{ \pm}^{1}}{\sim} z_{1}, \tilde{z}_{2} \stackrel{\tau, M_{ \pm}^{2}}{\sim} z_{2}\right\} \\
& =\inf \left\{\Phi\left[z_{1}, z_{2}\right]\left(\alpha_{1}, \alpha_{2}\right): \alpha_{1}, \alpha_{2} \in \mathscr{A}_{\tau}\right\} .
\end{aligned}
$$

Let us give a geometrical interpretation of the minimization problem

$$
\inf \left\{\Phi\left[z_{1}, z_{2}\right]\left(\alpha_{1}, \alpha_{2}\right): \alpha_{1}, \alpha_{2} \in \mathscr{A}_{\tau}\right\} \text {. }
$$

To this end, let $\alpha:[0, \tau] \rightarrow[0, \tau]^{2}$ be the parameterized path $\alpha(t):=\left(\alpha_{1}(t), \alpha_{2}(t)\right)$. Given a subset $\mathscr{S} \subset[0, \tau]^{2}$, denote by $\mathscr{T}_{\alpha}(\mathscr{S})$ the first time $\alpha$ hits $\mathscr{S}$ :

$$
\mathscr{T}_{\alpha}(\mathscr{S})=\inf \{t \in[0, \tau]: \alpha(t) \in \mathscr{S}\} .
$$

Set $\ell_{\left\{s_{i}=t\right\}}:=\left\{\left(s_{1}, s_{2}\right) \in[0, \tau]^{2}: s_{i}=t\right\}$, for $i=1,2$. By Lemma 3.11 and the continuity of $\alpha$,

$$
\begin{aligned}
\mathcal{T}_{z_{i} \circ \alpha_{i}}^{ \pm}=\left\{\alpha_{i}^{-1}(t): t \in \mathcal{T}_{z_{i}}^{ \pm}\right\} & =\left\{\inf \left\{s \in[0, \tau]: \alpha_{i}(s) \geq t\right\}: t \in \mathcal{T}_{z_{i}}^{ \pm}\right\} \\
& =\left\{\mathscr{T}_{\alpha}\left(\ell_{\left\{s_{i}=t\right\}}\right): t \in \mathcal{T}_{z_{i}}^{ \pm}\right\},
\end{aligned}
$$

for $i=1,2$. Similarly, $\kappa^{ \pm}=\mathscr{T}_{\alpha}\left(K^{ \pm}\right)$, where

$$
\begin{aligned}
& K^{+}=\left\{\left(s_{1}, s_{2}\right) \in[0, \tau]^{2}: \max \left\{\rho_{z_{1}}^{+}\left(s_{1}\right), \rho_{z_{2}}^{+}\left(s_{2}\right)\right\}>\max \left\{\frac{1}{M_{-}^{1}}, \frac{1}{M_{-}^{2}}\right\}\right\}, \\
& K^{-}=\left\{\left(s_{1}, s_{2}\right) \in[0, \tau]^{2}: \min \left\{\rho_{z_{1}}^{-}\left(s_{1}\right), \rho_{z_{2}}^{-}\left(s_{2}\right)\right\}<\min \left\{-\frac{1}{M_{+}^{1}},-\frac{1}{M_{+}^{2}}\right\}\right\} .
\end{aligned}
$$

Consequently,

$$
\begin{aligned}
\mathcal{T}_{z_{1} \circ \alpha_{1}}^{ \pm} \cup \mathcal{T}_{z_{2} \circ \alpha_{2}}^{ \pm} \cap\left(\kappa^{ \pm}, \tau\right]=\left\{\mathscr{T}_{\alpha}\left(\ell_{s_{1}=t} \cap K^{ \pm}\right):\right. & \left.t \in \mathcal{T}_{z_{1}}^{ \pm}\right\} \\
& \cup\left\{\mathscr{T}_{\alpha}\left(\ell_{s_{2}=t} \cap K^{ \pm}\right): t \in \mathcal{T}_{z_{2}}^{ \pm}\right\} .
\end{aligned}
$$

In other words, the value $\Phi\left[z_{1}, z_{2}\right]\left(\alpha_{1}, \alpha_{2}\right)$ is dependent only on where the path $\alpha$ hits $\mathcal{L}^{+} \cup \mathcal{L}^{-} ; \mathcal{L}^{ \pm}$are the line segments

$$
\mathcal{L}^{ \pm}:=\left\{\ell_{s_{1}=t} \cap K^{ \pm}: t \in \mathcal{T}_{z_{1}}^{ \pm}\right\} \cup\left\{\ell_{s_{2}=t} \cap K^{ \pm}: t \in \mathcal{T}_{z_{2}}^{ \pm}\right\},
$$

see Figure 3. As a result, $\Phi\left[z_{1}, z_{2}\right]\left(\alpha_{1}, \alpha_{2}\right)$ is a function of the path $\alpha$, independent of its parameterization.

From the view of factoring the solution operator, Theorem 2.10 is supplying a description of the metric induced by the uniform norm on the quotient space, cf. Figure 2 .

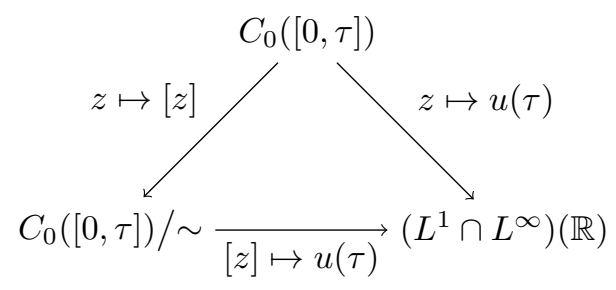

Figure 2. Schematic drawing of the factorization of the solution map. 
Based on the above observations, we now give an outline of how the minimization problem may be solved using dynamic programming. Introduce a cost function $c:[0, \tau]^{2} \rightarrow[0, \infty)$ by

$$
c(s)=\max \left\{\begin{array}{c}
\left|\rho_{z_{1}}^{+}\left(s_{1}\right)-\rho_{z_{2}}^{+}\left(s_{2}\right)\right| \mathbb{1}_{\mathcal{L}^{+}}(s), \\
\left|\rho_{z_{1}}^{-}\left(s_{1}\right)-\rho_{z_{2}}^{-}\left(s_{2}\right)\right| \mathbb{1}_{\mathcal{L}^{-}}(s), \\
\left|z_{1}(\tau)-z_{2}(\tau)\right| \mathbb{1}_{\{s=(\tau, \tau)\}}
\end{array}\right\},
$$

where $\mathbb{1}_{\mathscr{S}}$ is the characteristic function of $\mathscr{S}$. Hence,

$$
\Phi\left[z_{1}, z_{2}\right]\left(\alpha_{1}, \alpha_{2}\right)=\max \left\{c(\alpha(t)): t \in \cup_{i=1,2}\left\{\mathscr{T}_{\alpha}\left(\ell_{s_{i}=t}\right): t \in \mathcal{T}_{z_{i}}\right\} \cup\{\tau\}\right\} .
$$

For any $s \in[0, \tau]^{2}$, let $\mathcal{A}_{s}$ be the set of monotone paths connecting $s$ and $(\tau, \tau)$ :

$$
\mathcal{A}_{s}:=\left\{\alpha \in C\left([0,1] ;[0, \tau]^{2}\right): \begin{array}{l}
\alpha(0)=s, \alpha(1)=(\tau, \tau), \alpha=\left(\alpha_{1}, \alpha_{2}\right), \text { where } \\
\alpha_{1}, \alpha_{2} \text { are nondecreasing and continuous. }
\end{array}\right\} .
$$

Define a value function $V:[0, \tau]^{2} \rightarrow[0, \infty)$ by

$$
V(s)=\inf _{\alpha \in \mathcal{A}_{s}}\left\{\max \left\{c(\alpha(t)): t \in \cup_{i=1,2}\left\{\mathscr{T}_{\alpha}\left(\ell_{s_{i}=\tilde{t}}\right): \tilde{t} \in \mathcal{T}_{z_{i}}\right\} \cup\{1\}\right\}\right\},
$$

so that

$$
V((0,0))=\inf \left\{\Phi\left[z_{1}, z_{2}\right]\left(\alpha_{1}, \alpha_{2}\right): \alpha_{1}, \alpha_{2} \in \mathscr{A}_{\tau}\right\}
$$

is the sought value. Let us show how to compute $V$ on the grid

$$
\mathcal{G}=\left(\cup_{j} \ell_{s_{1}=\tau_{j}^{1}}\right) \cup\left(\cup_{k} \ell_{s_{2}=\tau_{k}^{2}}\right),
$$

where $\left\{\tau_{n}^{i}\right\}_{n=0}^{N_{i}}=\mathcal{T}_{z_{i}}$. First note that for $s \in \ell_{s_{1}=\tau}$, the set of admissible paths $\mathcal{A}_{s}$ is simply any path tracing out the straight line connecting $s$ and $(\tau, \tau)$. Hence,

$$
V\left(\left(\tau, s_{2}\right)\right)=\max \left\{c(\tau, t): t \in\left(\left\{s_{2}\right\} \cup \mathcal{T}_{z_{2}}\right) \cap\left[s_{2}, \tau\right]\right\}
$$

Similarly,

$$
V\left(\left(s_{1}, \tau\right)\right)=\max \left\{c(t, \tau): t \in\left(\left\{s_{1}\right\} \cup \mathcal{T}_{z_{1}}\right) \cap\left[s_{1}, \tau\right]\right\}
$$

To compute $V$ on the remaining part of $\mathcal{G}$, define the squares

$$
Q_{j, k}=\left[\tau_{j}^{1}, \tau_{j-1}^{1}\right] \times\left[\tau_{k}^{2}, \tau_{k-1}^{2}\right]
$$

and let

$$
\begin{aligned}
& \partial^{-} Q_{j, k}=\left\{\tau_{j}^{1}\right\} \times\left[\tau_{k}^{2}, \tau_{k-1}^{2}\right] \cup\left[\tau_{j}^{1}, \tau_{j-1}^{1}\right] \times\left\{\tau_{k}^{2}\right\}, \\
& \partial^{+} Q_{j, k}=\left\{\tau_{j-1}^{1}\right\} \times\left[\tau_{k}^{2}, \tau_{k-1}^{2}\right] \cup\left[\tau_{j}^{1}, \tau_{j-1}^{1}\right] \times\left\{\tau_{k-1}^{2}\right\} .
\end{aligned}
$$

Suppose $V$ is known on $\partial^{+} Q_{j, k}$. Then, for $s \in \partial^{-} Q_{j, k}$,

$$
V(s)=\max \left\{c(s), \min \left\{V(\tilde{s}): \tilde{s} \in \partial^{+} Q_{j, k} \cap\left\{\bar{s} \in[0, \tau]^{2}: \bar{s} \geq s\right\}\right\}\right\} .
$$

As $\partial^{+} Q_{j, k} \subset \partial^{-} Q_{j-1, k} \cup \partial^{-} Q_{j, k-1}$ for $j, k>1$ we may compute $V$ on the entire grid $\mathcal{G}$, starting in the upper right square $Q_{1,1}$ and trace our way down to the lower left square $Q_{N_{1}, N_{2}}$.

\section{Proofs of main Results}

In this section we provide detailed proofs of Theorems 2.4, 2.5, 2.8, and 2.10. 


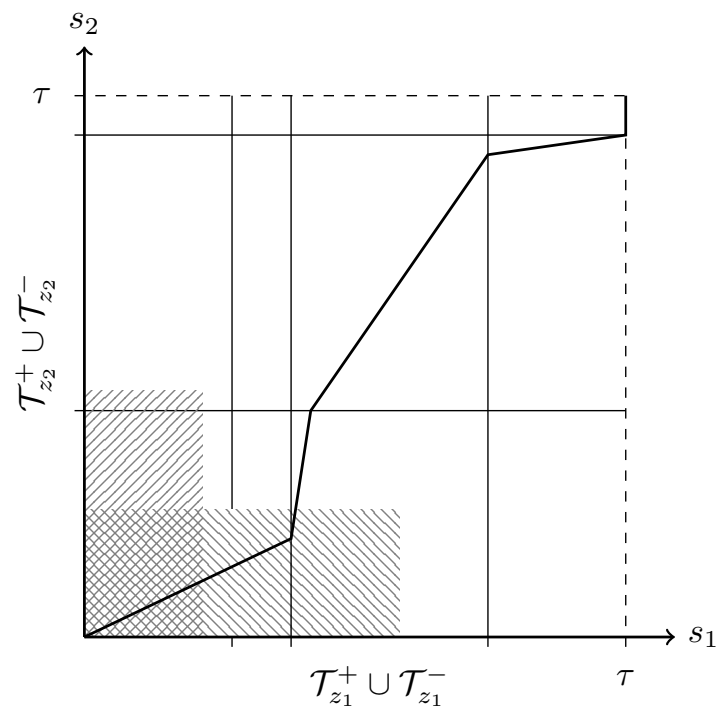

Figure 3. The curve $\alpha=\left(\alpha_{1}, \alpha_{2}\right)$ along with the line segments in $\mathcal{L}^{+}$and $\mathcal{L}^{-}$. The complement of $K^{+}$and $K^{-}$are hatched in grey.

3.1. Local solutions by substitution. For a given path $z \in C^{1}([0, T])$, let $v$ solve the conservation law

$$
\partial_{t} v+\partial_{x} f(v)=0, \quad v(z(0), x)=u_{0}(x)
$$

on $[z(0), \infty) \times \mathbb{R}$, and set $u(t, x):=v(z(t), x)$. Then, formally, it follows that $u$ solves (1.1). Let us take a closer look at this substitution, by considering the viscous approximation. That is, let $v^{\varepsilon}$ be the classical solution to the parabolic problem

$$
\partial_{t} v^{\varepsilon}+\partial_{x} f\left(v^{\varepsilon}\right)=\varepsilon \partial_{x}^{2} v^{\varepsilon}, \quad v^{\varepsilon}(z(0), x)=u_{0}(x)
$$

on $[z(0), \infty) \times \mathbb{R}$. Then $u^{\varepsilon}(t, x):=v^{\varepsilon}(z(t), x)$ satisfies, for any convex entropy, entropy-flux pair $(S, Q)$,

$$
\partial_{t} S\left(u^{\varepsilon}\right)+\partial_{x} Q\left(u^{\varepsilon}\right) \dot{z}=\varepsilon \partial_{x}^{2} S\left(u^{\varepsilon}\right) \dot{z}-\varepsilon S^{\prime \prime}\left(u^{\varepsilon}\right)\left(\partial_{x} u^{\varepsilon}\right)^{2} \dot{z}, \quad u^{\varepsilon}(0, x)=u_{0}(x) .
$$

A priori, due to the factor $\dot{z}$, the limiting solution does not necessarily dissipate the entropy. However, if $\partial_{x} v(z(t), x)=\partial_{x} u(t, x)$ is bounded, then the dissipation vanishes as $\varepsilon \rightarrow 0$ and $u$ ought to be a solution. Also, if $\dot{z} \geq 0$, then $u^{\varepsilon}$ ought to converge to the entropy solution to (1.1). Similarly, if $\dot{z} \leq 0$, we let $v^{\varepsilon}$ solve the parabolic problem with flux $-f$,

$$
\partial_{t} v^{\varepsilon}-\partial_{x} f\left(v^{\varepsilon}\right)=\varepsilon \partial_{x}^{2} v^{\varepsilon}, \quad v^{\varepsilon}(-z(0), x)=u_{0}(x)
$$

on $(-\infty,-z(0)) \times \mathbb{R}$, and take $u^{\varepsilon}(t, x)=u^{\varepsilon}(-z(t), x)$.

These observations are formalized in the next two lemmas. We consider first the case that $z$ is monotone.

Lemma 3.1. Assume that $f$ is Lipschitz continuous and $u_{0} \in\left(L^{\infty} \cap L^{1} \cap B V\right)(\mathbb{R})$. Suppose $z$ is Lipschitz continuous and nondecreasing on $[0, \tau]$ and $\tilde{z}$ is Lipschitz 
continuous on $[z(0), z(\tau)]$. Let $v$ be the entropy solution to

$$
\begin{cases}\partial_{t} v+\tilde{z}^{\prime} \partial_{x} f(v)=0, & (t, x) \in(z(0), z(\tau)) \times \mathbb{R} \\ v(z(0), x)=u_{0}(x) & x \in \mathbb{R}\end{cases}
$$

Set $u(t, x):=v(z(t), x)$. Then $u$ is an entropy solution to

$$
\begin{cases}\partial_{t} u+(\tilde{z} \circ z)^{\prime} \partial_{x} f(u)=0, & (t, x) \in(0, \tau) \times \mathbb{R}, \\ u(0, x)=u_{0}(x), & x \in \mathbb{R} .\end{cases}
$$

Proof. Assume first that $z$ is strictly increasing, i.e., $z^{\prime}>0$ on $(0, \tau)$, and set $z(0)=a, z(\tau)=b$. We need to show that $u(t, x)=v(z(t), x)$ satisfies

$$
\int_{0}^{\tau} \int_{\mathbb{R}} S(u) \partial_{t} \varphi+(\tilde{z} \circ z)^{\prime} Q(u) \partial_{x} \varphi d x d t+\int_{\mathbb{R}} S\left(u_{0}(x)\right) \varphi(0, x) d x \geq 0,
$$

for all convex entropy, entropy-flux pairs $(S, Q)$ and for all non-negative test function $\varphi \in C_{c}^{\infty}([0, \tau) \times \mathbb{R})$. Let $\phi(\zeta, x)=\varphi\left(z^{-1}(\zeta), x\right)$. Upon a change of variables it follows that

$$
\begin{aligned}
\int_{0}^{\tau} \int_{\mathbb{R}} S(u) \partial_{t} \varphi d x d t & =\int_{0}^{\tau} \int_{\mathbb{R}} S(v(z(t), x)) \partial_{z} \phi(z(t), x) \dot{z}(t) d x d t \\
& =\int_{a}^{b} \int_{\mathbb{R}} S(v(z, x)) \partial_{z} \phi(z, x) d x d z
\end{aligned}
$$

Similarly,

$$
\int_{0}^{\tau} \int_{\mathbb{R}}(\tilde{z} \circ z)^{\prime}(t) Q(u) \partial_{x} \varphi d x d t=\int_{a}^{b} \int_{\mathbb{R}} \tilde{z}^{\prime}(z) Q(v(z, x)) \partial_{x} \phi(z, x) d x d z,
$$

and

$$
\int_{\mathbb{R}} S\left(u_{0}(x)\right) \varphi(0, x) d x=\int_{\mathbb{R}} S(v(a, x)) \phi(a, x) d x .
$$

Hence, (3.1) follows due to the fact that $v$ is an entropy solution.

Next, suppose $z$ is merely nondecreasing, i.e., $z^{\prime} \geq 0$ on $(0, \tau)$, and $z(\tau)>z(0)$. Introduce the approximation

$$
z_{\varepsilon}(t)=(1-\varepsilon) z(t)+\varepsilon\left[\frac{z(\tau)-z(0)}{\tau} t+z(0)\right], \quad 0<\varepsilon<1,
$$

and note that $z_{\varepsilon}^{\prime}>0$. Take $u^{\varepsilon}(t, x)=v\left(z_{\varepsilon}(t), x\right)$ and send $\varepsilon \downarrow 0$ in 3.1.

If $z^{\prime} \equiv 0$ on $(0, \tau)$, then $\tilde{z}(t)=z(0)$ and $u(t, x)=v(z(t), x)=u_{0}(x)$, so (3.1) is satisfied.

In the following discussion it will be convenient for us to talk about backward entropy solutions. For us the natural backward solution is the (forward) entropy solution to the problem with flux $-f$. That is, the entropy solution to

$$
\partial_{t} v+\partial_{x} f(v)=0, \quad v(0, x)=v_{0}(x)
$$

on $(-\infty, 0] \times \mathbb{R}$ is obtained by solving the problem

$$
\partial_{t} w-\partial_{x} f(w)=0, \quad w(0, x)=v_{0}(x),
$$


on $[0, \infty) \times \mathbb{R}$ and setting $v(t, x)=w(-t, x)$. This yields the following backward Kružkov entropy condition for 3.2 on the interval $(-\infty, 0]$. For any convex entropy, entrop-flux pair $(S, Q)$, and for any non-negative $\varphi \in C_{c}^{\infty}((-\infty, 0] \times \mathbb{R})$,

$$
\int_{-\infty}^{0} \int_{\mathbb{R}} S(v) \partial_{t} \varphi+Q(v) \partial_{x} \varphi d x d t-\int_{\mathbb{R}} S\left(v_{0}(x)\right) \varphi(0, x) d x \leq 0 .
$$

By the one-to-one correspondence $v \mapsto w$ it is clear that the associated notion of backward entropy solution is well posed for any $u_{0} \in\left(L^{\infty} \cap L^{1}\right)(\mathbb{R})$. The backward/forward entropy solution to 3.2 on $(-\infty, \infty) \times \mathbb{R}$ is obtained by considering the forward solution for $t \geq 0$ and the backward solution for $t<0$. The fact that the initial condition was specified at time $t_{0}=0$ was somehow arbitrary, and the extension to general $t_{0} \in \mathbb{R}$ may be obtained by the substitution $t \mapsto t-t_{0}$.

Lemma 3.2. Suppose $u_{0} \in\left(L^{\infty} \cap L^{1} \cap B V\right)(\mathbb{R})$ and $f$ is Lipschitz continuous. Let $z$ be a Lipschitz continuous path on $[0, \tau]$. Write

$$
z_{\min }=\min _{0 \leq t \leq \tau}\{z(t)\} \quad \text { and } \quad z_{\max }=\max _{0 \leq t \leq \tau}\{z(t)\} .
$$

Let $u$ be the entropy solution to 1.1 on $[0, \tau]$ with initial condition $u_{0}$, and $v$ be the backward/forward entropy solution to

$$
\partial_{t} v+\partial_{x} f(v)=0, \quad v(z(0), x)=u_{0}(x),
$$

on $\left[z_{\min }, z_{\max }\right]$. Suppose $v$ is uniformly Lipschitz continuous in $x$ for any $t \in$ $\left[t_{1}, t_{2}\right] \subset\left(z_{\min }, z_{\max }\right)$. Then $u(t, x)=v(z(t), x)$. Furthermore $u$ is nondissipative, i.e., for any convex entropy, entropy-flux pair $(S, Q)$ and for any $\varphi \in C_{c}^{\infty}([0, \tau) \times \mathbb{R})$,

$$
\int_{0}^{\tau} \int_{\mathbb{R}} S(u) \partial_{t} \varphi+\dot{z} Q(u) \partial_{x} \varphi d x d t+\int_{\mathbb{R}} S\left(u_{0}(x)\right) \varphi(0, x) d x=0 .
$$

Proof. By the weak formulation of (3.3), using the Lipschitz continuity in $x$, for any $\left[t_{1}, t_{2}\right] \subset\left(z_{\min }, z_{\max }\right)$,

$$
\left|v\left(t_{2}, x\right)-v\left(t_{1}, x\right)\right| \leq \sup _{t_{1} \leq t \leq t_{2}}\left\{\left\|\partial_{x} f(v)\right\|_{L^{\infty}(\mathbb{R})}\right\}\left|t_{2}-t_{1}\right| .
$$

Consequently $v$ is locally Lipschitz continuous in time. Let us consider an approximation $\left\{z_{\varepsilon}\right\}_{\varepsilon>0} \subset C^{1}([0, \tau])$ of $z$ satisfying

$$
z_{\varepsilon} \rightarrow z \text { uniformly on }[0, \tau], \quad z_{\min }+\varepsilon \leq z_{\varepsilon} \leq z_{\max }-\varepsilon \text {. }
$$

Let $u^{\varepsilon}(t, x)=v\left(z_{\varepsilon}(t), x\right)$. As $t \mapsto v(t)$ is $L^{1}$-Lipschitz continuous, $u_{\varepsilon} \rightarrow u$ in $C\left([0, \tau] ; L^{1}(\mathbb{R})\right)$. Using integration by parts and the chain rule [1, Theorem 3.99] we obtain

$$
\begin{aligned}
\int_{0}^{\tau} & \int_{\mathbb{R}} S(u) \partial_{t} \varphi+\dot{z} Q(u) \partial_{x} \varphi d x d t+\int_{\mathbb{R}} S\left(u_{0}(x)\right) \varphi(0, x) d x \\
& =\lim _{\varepsilon \downarrow 0} \int_{0}^{\tau} \int_{\mathbb{R}} S\left(u^{\varepsilon}\right) \partial_{t} \varphi+\dot{z}_{\varepsilon} Q\left(u^{\varepsilon}\right) \partial_{x} \varphi d x d t+\int_{\mathbb{R}} S\left(v\left(z_{\varepsilon}(0), x\right)\right) \varphi(0, x) d x \\
& =-\lim _{\varepsilon \downarrow 0} \int_{0}^{\tau} \int_{\mathbb{R}} \underbrace{\left[\partial_{z} S\left(v\left(z_{\varepsilon}(t), x\right)\right)+\partial_{x} Q\left(v\left(z_{\varepsilon}(t), x\right)\right)\right]}_{m_{v}\left(z_{\varepsilon}(t), x\right)} \dot{z}_{\varepsilon}(t) \varphi(t, x) d x d t .
\end{aligned}
$$

By Lipschitz continuity, $m_{v} \in L^{\infty}\left(\left[z_{\min }+\varepsilon, z_{\max }-\varepsilon\right] \times \mathbb{R}\right)$ for $\varepsilon>0$. Moreover, by the chain-rule, $m_{v}(z, x)=0$ for almost all $(z, x) \in\left[z_{\min }, z_{\max }\right] \times \mathbb{R}$. Hence, we conclude that 3.4 holds. 
3.2. Spatial regularity estimates for piecewise linear paths. Let $u$ be the entropy solution to (1.1) with path $z$. In view of Lemma 3.2 , it is necessary to keep track of the best possible bounds $t \mapsto\left(-M_{-}(t), M_{+}(t)\right)$ satisfying

$$
-M_{-}(t) \leq \frac{f^{\prime}(u(t, y))-f^{\prime}(u(t, x))}{y-x} \leq M_{+}(t), \quad x, y \in \mathbb{R}, x<y .
$$

We begin with the following Oleĭnik-type estimate:

Lemma 3.3. Suppose $\left(\mathcal{A}_{f}\right)$ holds and $u_{0} \in L^{\infty}(\mathbb{R}) \cap B V(\mathbb{R})$ satisfies 2.1. Fix $\lambda \in \mathbb{R}$ and let $u$ be the entropy solution to

$$
\partial_{t} u+\lambda \partial_{x} f(u)=0, \quad u(0, x)=u_{0}(x),
$$

for some constant $\lambda$. Let $\Theta(x)=x$ for $x \geq 0$ and $\Theta(x)=\infty$ for $x<0$. Then

$$
-\Theta\left(\frac{1}{\left(M_{-}\right)^{-1}-t \lambda}\right) \leq \frac{f^{\prime}(u(t, y \pm))-f^{\prime}(u(t, x \pm))}{y-x} \leq \Theta\left(\frac{1}{\left(M_{+}\right)^{-1}+t \lambda}\right)
$$

for all $-\infty<x<y<\infty$. Whenever $M_{ \pm}$takes the values 0 or $\infty$, we use the convention $(\infty)^{-1}=0$ and $(0)^{-1}=\infty$.

Proof. Let $\xi_{ \pm}$be the maximal/minimal backward characteristic emanating from $(t, x)$ and $\zeta_{ \pm}$be the maximal/minimal backward characteristic emanating from $(t, y)$ on $[0, t]$, cf. [12, $\S 10.2]$. By [12, Theorem 10.3.2], both $\xi_{ \pm}$and $\zeta_{ \pm}$are shock free on $[\tau, t]$. In view of [12, Theorem 11.1.1],

$$
x=\xi_{ \pm}(0)+t \lambda f^{\prime}(u(t, x \pm)), \quad \text { and } y=\zeta_{ \pm}(0)+t \lambda f^{\prime}(u(t, y \pm)) .
$$

where $\xi_{ \pm}(0) \leq \zeta_{ \pm}(0)$. Note, these equalities are true when we chose either + or - . That is, when considering $x+$, we apply $\xi_{+}$and so forth. The case $\lambda=0$ is trivial. We consider the cases $\lambda>0$ and $\lambda<0$ separately.

Assume $\lambda>0$. Consider the upper bound in (3.5). Assume $f^{\prime}(u(t, y \pm))-$ $f^{\prime}(u(t, x \pm))>0$. It follows that

$$
\frac{y-x}{f^{\prime}(u(t, y \pm))-f^{\prime}(u(t, x \pm))}=\frac{\zeta_{ \pm}(0)-\xi_{ \pm}(0)}{f^{\prime}(u(t, y \pm))-f^{\prime}(u(t, x \pm))}+t \lambda .
$$

By [12, Theorem 11.1.3],

$$
\left\{\begin{array}{l}
u_{0}\left(\zeta_{ \pm}(0)-\right) \leq u(t, y \pm) \leq u_{0}\left(\zeta_{ \pm}(0)+\right) \\
u_{0}\left(\xi_{ \pm}(0)-\right) \leq u(t, x \pm) \leq u_{0}\left(\xi_{ \pm}(0)+\right)
\end{array}\right.
$$

Hence, since $f^{\prime}$ is increasing,

$$
0<f^{\prime}(u(t, y \pm))-f^{\prime}(u(t, x \pm)) \leq f^{\prime}\left(u_{0}\left(\zeta_{ \pm}(0)+\right)\right)-f^{\prime}\left(u_{0}\left(\xi_{ \pm}(0)-\right)\right) .
$$

If $M_{+}=0$ this cannot be true, and so $f^{\prime}(u(t, y \pm))-f^{\prime}(u(t, x \pm)) \leq 0$, which proves the upper bound for $M_{+}=0$. Assume $M_{+}>0$. Then, by the above,

$$
\begin{aligned}
\frac{y-x}{f^{\prime}(u(t, y \pm))-f^{\prime}(u(t, x \pm)} & \geq \frac{\zeta_{ \pm}(0)-\xi_{ \pm}(0)}{\left.f^{\prime}\left(u_{0}\left(\zeta_{ \pm}(0)+\right)\right)\right)-f^{\prime}\left(u_{0}\left(\xi_{ \pm}(0)-\right)\right)}+t \lambda \\
& \geq \frac{1}{M_{+}}+t \lambda .
\end{aligned}
$$

Consider the lower bound in 3.5. Assume $f^{\prime}(u(t, y \pm))-f^{\prime}(u(t, x \pm))<0$. Arguing as above,

$$
0>f^{\prime}(u(t, y \pm))-f^{\prime}(u(t, x \pm)) \geq f^{\prime}\left(u_{0}\left(\zeta_{ \pm}(0)-\right)\right)-f^{\prime}\left(u_{0}\left(\xi_{ \pm}(0)+\right)\right) .
$$


If $M_{-}=0$, this yields a contradiction, and so $f^{\prime}(u(t, y \pm))-f^{\prime}(u(t, x \pm)) \geq 0$, proving the lower bound for $M_{-}=0$. Assume $M_{-}>0$. Inserting (3.7) into 3.6 yields

$$
\begin{aligned}
\frac{y-x}{f^{\prime}(u(t, y \pm))-f^{\prime}(u(t, x \pm)} & \leq \frac{\zeta_{ \pm}(0)-\xi_{ \pm}(0)}{f^{\prime}\left(u_{0}\left(\zeta_{ \pm}(0)+\right)\right)-f^{\prime}\left(u_{0}\left(\xi_{ \pm}(0)-\right)\right)}+t \lambda \\
& \leq-\frac{1}{M_{-}}+t \lambda .
\end{aligned}
$$

Assuming $t \lambda<\left(M_{-}\right)^{-1}$ yields the lower bound in (3.5).

Assume $\lambda<0$. By [12, Theorem 11.1.3] (upon reversing the inequality as $\lambda f$ is concave) we have

$$
\left\{\begin{array}{l}
u_{0}\left(\zeta_{ \pm}(0)+\right) \leq u(t, y \pm) \leq u_{0}\left(\zeta_{ \pm}(0)-\right), \\
u_{0}\left(\xi_{ \pm}(0+) \leq u(t, x \pm) \leq u_{0}\left(\xi_{ \pm}(0)-\right)\right.
\end{array}\right.
$$

Consider the upper bound in (3.5). Assume $f^{\prime}(u(t, y \pm))-f^{\prime}(u(t, x \pm))>0$. Then

$$
0<f^{\prime}(u(t, y \pm))-f^{\prime}(u(t, x \pm)) \leq f^{\prime}\left(u_{0}\left(\zeta_{ \pm}(0)-\right)\right)-f^{\prime}\left(u_{0}\left(\xi_{ \pm}(0)+\right)\right)
$$

If $M_{+}=0$ we obtain a contradiction. Assume $M_{+}>0$. Inserting into (3.6) yields

$$
\begin{aligned}
\frac{y-x}{f^{\prime}(u(t, y \pm))-f^{\prime}(u(t, x \pm)} & \geq \frac{\zeta_{ \pm}(0)-\xi_{ \pm}(0)}{\left.f^{\prime}\left(u_{0}\left(\zeta_{ \pm}(0)-\right)\right)\right)-f^{\prime}\left(u_{0}\left(\xi_{ \pm}(0)+\right)\right)}+t \lambda \\
& \geq \frac{1}{M_{+}}+t \lambda .
\end{aligned}
$$

Assuming $-t \lambda<\left(M_{+}\right)^{-1}$ yields the upper bound in 3.5 . Consider the lower bound in (3.5). Assume $f^{\prime}(u(t, y \pm))-f^{\prime}(u(t, x \pm))<0$. By (3.8),

$$
0>f^{\prime}(u(t, y \pm))-f^{\prime}(u(t, x \pm)) \geq f^{\prime}\left(u_{0}\left(\zeta_{ \pm}(0)+\right)\right)-f^{\prime}\left(u_{0}\left(\xi_{ \pm}(0)-\right)\right) .
$$

If $M_{-}=0$ we obtain a contradiction. Assume $M_{-}>0$. Inserting into $\sqrt{3.6}$ yields

$$
\begin{aligned}
\frac{y-x}{f^{\prime}(u(t, y \pm))-f^{\prime}(u(t, x \pm)} & \leq \frac{\zeta_{ \pm}(0)-\xi_{ \pm}(0)}{f^{\prime}\left(u_{0}\left(\zeta_{ \pm}(0)+\right)\right)-f^{\prime}\left(u_{0}\left(\xi_{ \pm}(0)-\right)\right)}+t \lambda \\
& \leq-\frac{1}{M_{-}}+t \lambda
\end{aligned}
$$

This yields the lower bound for 3.5 .

Combining Lemmas 3.2 and 3.3 we obtain

Corollary 3.4. Suppose $\left(\mathcal{A}_{f}\right)$ holds, and that $u_{0} \in\left(L^{\infty} \cap L^{1} \cap B V\right)(\mathbb{R})$ satisfies (2.1) for some $0 \leq M_{-}, M_{+} \leq \infty$. Let $z$ be a Lipschitz continuous path, satisfying

$$
z(0)=0, \quad-\left(M_{+}\right)^{-1} \leq z(t) \leq\left(M_{-}\right)^{-1}, \quad t \in[0, \tau],
$$

for some $\tau>0$. Let $v$ be the backward/forward entropy solution to

$$
\partial_{t} v+\partial_{x} f(v)=0, \quad v(0, x)=u_{0}(x),
$$

on $\left[-\left(M_{+}\right)^{-1},\left(M_{-}\right)^{-1}\right]$. We apply the convention $(0)^{-1}=\infty$ and $(\infty)^{-1}=0$. Then $u(t, x):=v(z(t), x)$ is a nondissipative entropy solution to 1.1 on $[0, \tau]$.

Proof. Recall that $v$ is composed of a backward and a forward solution. That is,

$$
v(t, x):= \begin{cases}w_{+}(t, x) & t \in\left[0,\left(M_{-}\right)^{-1}\right), \\ w_{-}(-t, x) & t \in\left(-\left(M_{+}\right)^{-1}, 0\right)\end{cases}
$$


where $w_{ \pm}$are the entropy solutions to

$$
\left\{\begin{array}{lll}
\partial_{t} w_{+}+\partial_{x} f\left(w_{+}\right)=0, & t \in\left(0,\left(M_{-}\right)^{-1}\right), & w_{+}(0, x)=u_{0}(x) \\
\partial_{t} w_{-}-\partial_{x} f\left(w_{-}\right)=0, & t \in\left(0,\left(M_{+}\right)^{-1}\right), & w_{-}(0, x)=u_{0}(x)
\end{array}\right.
$$

Consequently, by Lemma 3.3 .

$$
-\frac{1}{\left(M_{-}\right)^{-1}-t} \leq \frac{f^{\prime}(v(t, y \pm))-f^{\prime}(v(t, x \pm))}{y-x} \leq \frac{1}{\left(M_{+}\right)^{-1}+t}
$$

The result follows from Lemma 3.2

We are now ready to prove Theorem 2.5 for piecewise linear paths. However, it will be convenient for us to apply Lemma 2.1. so we begin with its proof.

Proof of Lemma 2.1. (i). Suppose $t \in \mathscr{B}_{z}^{+}, t>0$. The argument for $\mathscr{B}_{z}^{-}$is analogous. By definition $\rho_{z}^{+}(t) \geq z(t)$. To arrive at a contradiction, suppose $\rho_{z}^{+}(t)>z(t)$. By continuity of $\rho_{z}^{+}$, there exits $\delta>0$ such that $\rho_{z}^{+}(s)>\rho_{z}^{+}(t)$ for all $0<t-s<\delta$, contradicting the fact that inf $\left\{s \in[0, T]: \rho_{z}^{+}(s) \geq \rho_{z}^{+}(t)\right\}=t$. Suppose $t \in \mathscr{B}_{z}^{-} \cap \mathscr{B}_{z}^{+}, t>0$. Then, by the above, $\rho_{z}^{-}(t)=z(t)=\rho_{z}^{+}(t)$, which can only be true if $z(t)=0$. Consequently

$$
\inf \left\{s \in[0, T]: \rho_{z}^{+}(s) \geq \rho_{z}^{+}(t)\right\}=0,
$$

contradicting the fact that $t \in \mathscr{B}_{z}^{+} \backslash\{0\}$.

(ii). Assume that $t_{n} \uparrow t$ where $\left\{t_{n}\right\}_{n \geq 1} \subset \mathscr{B}_{z}^{+}$. We need to show that $t \in \mathscr{B}_{z}^{+}$. As $t_{n} \in \mathscr{B}_{z}^{+}$

$$
\left\{s \in[0, T]: \rho_{z}^{+}(s) \geq \rho_{z}^{+}\left(t_{n}\right)\right\}=\left[t_{n}, T\right],
$$

for all $n \geq 1$. Consequently, as $\rho_{z}^{+}\left(t_{n}\right) \uparrow \rho_{z}^{+}(t)$,

$$
\inf \left\{s \in[0, T]: \rho_{z}^{+}(s) \geq \rho_{z}^{+}(t)\right\}=\inf \left(\bigcap_{n \geq 1}\left[t_{n}, T\right]\right)=t,
$$

showing that $t \in \mathscr{B}_{z}^{+}$. For the second statement, let $t=\sup \left\{\mathscr{B}_{z}^{ \pm} \cap[0, \tau]\right\}$. By definition of the supremum, there exists an increasing sequence $\left\{t_{n}\right\}_{n \geq 1} \subset \mathscr{B}_{z}^{+} \cap$ $[0, \tau]$ such that $t_{n} \uparrow t$.

(iii). Suppose $\left(t_{1}, t_{2}\right] \cap \mathscr{B}_{z}^{+}=\emptyset$. Then $\rho_{z}^{+}$is constant on $\left[t_{1}, t_{2}\right]$. The proof for $\mathscr{B}_{z}^{-}, \rho_{z}^{-}$is analogous. Assume there exist $s_{1}$ and $s_{2}$ with $t_{1}<s_{1}<s_{2} \leq t_{2}$ such that $\rho_{z}^{+}\left(s_{2}\right)>\rho_{z}^{+}\left(s_{1}\right)$. Then

$$
\rho_{z}^{+}\left(s_{2}\right)-\rho_{z}^{+}\left(s_{1}\right)>2 \varepsilon\left(s_{2}-s_{1}\right) \text { for some } \varepsilon>0 .
$$

Let

$$
t^{*}=\inf \left\{s \in\left[s_{1}, s_{2}\right]: \rho_{z}^{+}\left(s_{2}\right)-\rho_{z}^{+}(s) \leq \varepsilon\left(s_{2}-s\right)\right\} .
$$

By continuity of $\rho_{z}^{+}$it follows that $s_{1}<t^{*}<s_{2}$. As $t^{*} \notin \mathscr{B}_{z}^{+}$it follows by (i) that

$$
\inf \left\{s \in[0, T]: \rho_{z}^{+}(s) \geq \rho_{z}^{+}\left(t^{*}\right)\right\}=\hat{t}<t^{*} .
$$

Since $\rho^{+}$is nondecreasing, $\rho^{+}(\hat{t})=\rho^{+}\left(t^{*}\right)$, and $\rho^{+}$is constant on $\left[\hat{t}, t^{*}\right]$. But then, for any $\hat{t} \leq s \leq t^{*}$,

$$
\rho_{z}^{+}\left(s_{2}\right)-\rho_{z}^{+}(s)=\rho_{z}^{+}\left(s_{2}\right)-\rho_{z}^{+}\left(t^{*}\right)=\varepsilon\left(s_{2}-t^{*}\right) \leq \varepsilon\left(s_{2}-s\right),
$$

contradicting the definition of $t^{*}$.

(iv). We first prove the following claim: If $\partial_{-} \rho_{z}^{ \pm}(t)=0$ for all $t \in\left(t_{1}, t_{2}\right]$, then $\rho_{z}^{ \pm}$is constant on $\left[t_{1}, t_{2}\right]$. Let $s_{1}, s_{2}, t^{*}$ and $\varepsilon$ be as in the proof of (iii), and we 
also assume that $\rho^{+}\left(s_{2}\right)>\rho^{+}\left(s_{1}\right)$. By assumption $\partial_{-} \rho_{z}^{+}\left(t^{*}\right)=0$, so there exists a $\delta>0$ such that

$$
\rho_{z}^{+}\left(t^{*}\right)-\rho_{z}^{+}(s) \leq \varepsilon\left(t^{*}-s\right) \quad \text { for all } \quad t^{*}-\delta<s<t^{*} .
$$

But then, for any $t^{*}-\delta<s<t^{*}$,

$$
\rho_{z}^{+}\left(s_{2}\right)-\rho_{z}^{+}(s)=\underbrace{\rho_{z}^{+}\left(s_{2}\right)-\rho_{z}^{+}\left(t^{*}\right)}_{=\varepsilon\left(s_{2}-t^{*}\right)}+\underbrace{\rho_{z}^{+}\left(t^{*}\right)-\rho_{z}^{+}(s)}_{\leq \varepsilon\left(t^{*}-s\right)} \leq \varepsilon\left(s_{2}-s\right),
$$

contradicting the definition of $t^{*}$, thus finishing the proof of the claim. Consider the statement for $\mathscr{B}_{z}^{+}$. Let

$$
\mathscr{C}^{+}:=\left\{t \in(0, T]: \partial_{-} \rho_{z}^{+}(t)>0\right\} \cup\{0\} .
$$

Let $t \in \mathscr{B}_{z}^{+} \backslash\{0\}$. Suppose there exits $\delta>0$ such that for all $0<t-s<\delta$, $\partial_{-} \rho_{z}^{+}(s)=0$. Then, as $\rho_{z}^{+}$is constant on $(t-\delta, t)$, this contradicts the fact that $t \in \mathscr{B}_{z}^{+} \backslash\{0\}$. Consequently, there exists a sequence $t_{n} \uparrow t$ as $n \rightarrow \infty$ with $t_{n} \in \mathscr{C}^{+}$ for all $n \geq 1$. Hence $\mathscr{B}_{z}^{+} \subset \mathrm{cl}_{-}\left(\mathscr{C}^{+}\right)$. Next, suppose that $t \in \mathscr{C}^{+} \backslash\{0\}$. As $\partial_{-} \rho_{z}^{+}(t)>0$ it follows that $\rho_{z}^{+}(s)<\rho_{z}^{+}(t)$ for all $s<t$. Hence,

$$
\inf \left\{s \in[0, T]: \rho_{z}^{+}(s) \geq \rho_{z}^{+}(t)\right\}=t,
$$

so that $\mathscr{C}^{+} \subset \mathscr{B}_{z}^{+}$by (i). We then conclude, by (ii), that $\mathrm{cl}_{-}\left(\mathscr{C}^{+}\right) \subset \mathscr{B}_{z}^{+}$. The proof for $\mathscr{B}_{z}^{-}$is analogous.

Lemma 3.5. Suppose $\left(\mathcal{A}_{f}\right)$ holds and $u_{0} \in\left(L^{\infty} \cap L^{1} \cap B V\right)(\mathbb{R})$ satisfies $(2.1)$. Fix a piecewise linear path $z(t)$ with $z(0)=0$. Let $u$ be the corresponding entropy solution to (1.1), and $\rho_{z}^{+}, \rho_{z}^{-}$be defined by 2.2. Then, for any $t \in[0, T]$,

$$
-\frac{1}{\rho_{z}^{+}(t)-z(t)} \leq \frac{f^{\prime}(u(t, y \pm))-f^{\prime}(u(t, x \pm))}{y-x} \leq \frac{1}{z(t)-\rho_{z}^{-}(t)}, \quad x, y \in \mathbb{R}, x<y .
$$

We apply the convention $(0)^{-1}=\infty$.

Proof. Let $0 \leq t \leq T$ be given. By assumption there exists a finite sequence $\left\{t_{n}\right\}_{n=0}^{N}, 0=t_{0}<\cdots<t_{N}=T$, such that the graph of $z$ is a straight line on each interval $\left[t_{n}, t_{n+1}\right], n=0, \ldots, N-1$. Without loss of generality, we will prove the result for $t \in\left\{t_{n}\right\}_{n=0}^{N}$. Let $P_{n}$ be the statement of the lemma for $t=t_{n}$. We need to prove that $P_{n+1}$ holds given the validity of $P_{n}$, where $P_{n}$ precisely reads

$$
-\underbrace{\frac{1}{\rho_{z}^{+}\left(t_{n}\right)-z\left(t_{n}\right)}}_{M_{-}^{n}} \leq \underbrace{\frac{f^{\prime}\left(u\left(t_{n}, y \pm\right)\right)-f^{\prime}\left(u\left(t_{n}, x \pm\right)\right)}{y-x}}_{X_{n}} \leq \underbrace{\frac{1}{z\left(t_{n}\right)-\rho_{z}^{-}\left(t_{n}\right)}}_{M_{+}^{n}}, \quad x<y .
$$

By Lemma 3.3 ,

$$
-\Theta\left(\frac{1}{\left(M_{-}^{n}\right)^{-1}-\Delta z_{n}}\right) \leq X_{n+1} \leq \Theta\left(\frac{1}{\left(M_{+}^{n}\right)^{-1}+\Delta z_{n}}\right),
$$

where $\Delta z_{n}=z\left(t_{n+1}\right)-z\left(t_{n}\right)$. Therefore,

$$
-\Theta\left(\frac{1}{\rho_{z}^{+}\left(t_{n}\right)-z\left(t_{n+1}\right)}\right) \leq X_{n+1} \leq \Theta\left(\frac{1}{z\left(t_{n+1}\right)-\rho_{z}^{-}\left(t_{n}\right)}\right) .
$$

We consider three different cases:

$$
\text { (i) }: t_{n+1} \in \mathscr{B}_{z}^{+}, \quad \text { (ii) }: t_{n+1} \in \mathscr{B}_{z}^{-}, \quad \text { (iii) }: t_{n+1} \notin \mathscr{B}_{z}^{+} \cup \mathscr{B}_{z}^{-} \text {. }
$$


Consider (i). By Lemma 2.1 (i), $z\left(t_{n+1}\right)=\rho_{z}^{+}\left(t_{n+1}\right)$. If $\left(t_{n}, t_{n+1}\right] \cap \mathscr{B}_{z}^{-} \neq \emptyset$, then $t_{n+1} \in \mathscr{B}_{z}^{-}$by Lemma 2.1 (iv), but this cannot be the case as $\mathscr{B}_{z}^{+} \cap \mathscr{B}_{z}^{-}=\{0\}$. Consequently, by Lemma 2.1 (iii), $\rho_{z}^{-}\left(t_{n}\right)=\rho_{z}^{-}\left(t_{n+1}\right)$. Hence $P_{n+1}$ follows in case (i). Case (ii) is analogous. In case (iii), we argue as in case (i) to conclude that $\rho_{z}^{ \pm}\left(t_{n}\right)=\rho_{z}^{ \pm}\left(t_{n+1}\right)$. This proves $P_{n+1}$ in case (iii). It remains to observe that $P_{0}$ holds by assumption (2.1).

3.3. Equivalence. Let us first, for convenience, collect some consequences of the above results in terms of the equivalence relation, see Definition 2.3 .

Corollary 3.6. Assume that $u_{0} \in\left(L^{\infty} \cap L^{1} \cap B V\right)(\mathbb{R})$. Let $z_{1}$ and $z_{2}$ be Lipschitz continuous paths on $\left[t_{1}, t_{2}\right]$, and set $\Delta z_{i}=z_{i}\left(t_{2}\right)-z_{i}\left(t_{1}\right), i=1,2$. Then the following three statements hold:

(1) If $\Delta z_{1}=\Delta z_{2}$ and both paths are monotone. Then $z_{1} \sim z_{2}$ on $\left[t_{1}, t_{2}\right]$ with initial condition $u_{0}$.

(2) If $z_{1}=z_{2} \circ \alpha$ for some nondecreasing, Lipschitz continuous, surjective map $\alpha:\left[t_{1}, t_{2}\right] \rightarrow\left[t_{1}, t_{2}\right]$, then $z_{1} \sim z_{2}$ on $\left[t_{1}, t_{2}\right]$ with initial condition $u_{0}$.

(3) If $u_{0}$ satisfies 2.1) and

$$
-\left(M_{+}\right)^{-1} \leq z_{i}(t)-z_{i}\left(t_{1}\right) \leq\left(M_{-}\right)^{-1}, \text { for all } t_{1} \leq t \leq t_{2},
$$

and $\Delta z_{1}=\Delta z_{2}$, then $z_{1} \sim z_{2}$ on $\left[t_{1}, t_{2}\right]$ with initial condition $u_{0}$.

Proof. Let $u^{1}, u^{2}$ denote the entropy solutions to (1.1) associated with the paths $z_{1}, z_{2}$ and initial condition $u_{0}$.

(1). Suppose $z_{1}$ and $z_{2}$ are nondecreasing. We need to show that $u^{1}\left(t_{2}\right)=u^{2}\left(t_{2}\right)$. To apply Lemma 3.1. assume for notational ease $\left[t_{1}, t_{2}\right]=[0, \tau]$. Let $\tilde{z}(t)=t$ in Lemma 3.1. Then $u^{i}(t, x)=v\left(z_{i}(t), x\right), i=1,2$, where $v$ is the entropy solution to

$$
\partial_{t} v+\partial_{x} f(v)=0, \quad v(z(0), x)=u_{0}(x) .
$$

It follows that $u^{1}(\tau)=v\left(z_{1}(\tau)\right)=v\left(z_{2}(\tau)\right)=u^{2}(\tau)$. If $z_{1}, z_{2}$ are nonincreasing, we consider instead $\tilde{z}(t)=-t$ and the paths $-z_{1},-z_{2}$, and proceed as above.

(2). By Lemma 3.1, $u(t, x):=u^{2}(\alpha(t), x)$ satisfies

$$
\partial_{t} u+\left(z_{2} \circ \alpha\right)^{\prime} \partial_{x} f(u)=0, \quad u(0, x)=u^{2}\left(\alpha\left(t_{1}\right), x\right)=u_{0}(x) .
$$

But then $u=u^{1}$, and so $u^{1}\left(t_{2}\right)=u^{2}\left(\alpha\left(t_{2}\right)\right)=u^{2}\left(t_{2}\right)$.

(3). Set $\tilde{z}_{i}(t)=z_{i}\left(t+t_{1}\right)-z_{i}\left(t_{1}\right)$, and apply Corollary 3.4 with $\tilde{z}_{i}, i=1,2$. This yields

$$
u^{1}\left(t_{2}\right)=\tilde{u}^{1}\left(t_{2}-t_{1}\right)=v\left(\tilde{z}_{1}\left(t_{2}-t_{1}\right)\right)=v\left(\tilde{z}_{2}\left(t_{2}-t_{1}\right)\right)=\tilde{u}^{2}\left(t_{2}-t_{1}\right)=u^{2}\left(t_{2}\right),
$$

where $\tilde{u}^{1}$ and $\tilde{u}^{2}$ are the entropy solutions to 1.1 with $z=\tilde{z}_{1}$ and $z=\tilde{z}_{2}$, respectively.

Next we provide preliminary version of Theorem 2.8 .

Lemma 3.7. Suppose $f$ satisfies $\left(\mathcal{A}_{f}\right)$. Let $z_{1}, z_{2}$ be piecewise linear, continuous paths on $[0, \tau]$, satisfying $z_{1}(0)=z_{2}(0)=0$ and $z_{1}(\tau)=z_{2}(\tau)$. Suppose there exist piecewise linear nondecreasing surjective maps $\alpha_{i}:[0, \tau] \rightarrow[0, \tau], i=1,2$, such that $\rho_{z_{1}}^{ \pm} \circ \alpha_{1}=\rho_{z_{2}}^{ \pm} \circ \alpha_{2}$. Let $u^{1}$ and $u^{2}$ be entropy solutions to 1.1 with initial condition $u_{0} \in\left(L^{\infty} \cap L^{1} \cap B V\right)(\mathbb{R})$ satisfying $(2.1)$. Then $u^{1}(\tau)=u^{2}(\tau)$. That is, $z_{1} \sim z_{2}$ on $[0, \tau]$ for all $u_{0} \in\left(L^{\infty} \cap L^{1} \cap B V\right)(\mathbb{R})$ satisfying 2.1 . 
Proof. Set $\tilde{z}_{i}=z_{i} \circ \alpha_{i}, i=1,2$. By Corollary $3.6(2), \tilde{z}_{i} \sim z_{i}$. Furthermore, $\tilde{z}_{i}$ is piecewise linear with $\tilde{z}_{i}(0)=0$, and $\tilde{z}_{1}(\tau)=\tilde{z}_{2}(\tau)$. Hence, we might as well assume $\rho_{z_{1}}^{ \pm}=\rho_{z_{2}}^{ \pm}=\rho^{ \pm}$which also implies $\mathscr{B}_{z_{1}}^{ \pm}=\mathscr{B}_{z_{2}}^{ \pm}=: \mathscr{B}^{ \pm}$.

By Lemma 2.1 (iv),

$$
\mathscr{B}^{-} \cup \mathscr{B}^{+} \backslash\{0\}=\bigcup_{i=1}^{N}\left(s_{i}, t_{i}\right], \quad t_{0}=0 \leq s_{1}<t_{1}<\cdots<s_{N}<t_{N} \leq \tau=s_{N+1},
$$

for some $0 \leq N<\infty$. Suppose $u^{1}\left(t_{n}\right)=u^{2}\left(t_{n}\right)$. We want to show that $u^{1}\left(s_{n+1}\right)=$ $u^{2}\left(s_{n+1}\right)$. Assume $t_{n}<\tau$, for otherwise $t_{n}=\tau=s_{n+1}$ and we are done. Then $\left(t_{n}, s_{n+1}\right] \cap\left(\mathscr{B}^{-} \cup \mathscr{B}^{+}\right)=\emptyset$. By Lemma 2.1 (iii),

$$
\rho^{-}\left(t_{n}\right) \leq z_{1}(t), z_{2}(t) \leq \rho^{+}\left(t_{n}\right)
$$

for all $t \in\left[t_{n}, s_{n+1}\right]$. Moreover, due to Lemma 2.1 (i), $z_{1}\left(t_{n}\right)=z_{2}\left(t_{n}\right)=: a$ and $z_{1}\left(s_{n+1}\right)=z_{2}\left(s_{n+1}\right)$. By Lemma 3.5 .

$$
-\underbrace{\frac{1}{\rho^{+}\left(t_{n}\right)-a}}_{M_{-}} \leq \frac{f^{\prime}\left(u\left(t_{n}, y \pm\right)\right)-f^{\prime}\left(u\left(t_{n}, x \pm\right)\right)}{y-x} \leq \underbrace{\frac{1}{a-\rho^{-}\left(t_{n}\right)}}_{M_{+}}, \quad x<y .
$$

By Corollary 3.6 (3), since $z_{1}$ and $z_{2}$ satisfy (3.11), it follows that $z_{1} \sim z_{2}$ on $\left[t_{n}, s_{n+1}\right]$, and so $u^{1}\left(s_{n+1}\right)=u^{2}\left(s_{n+1}\right)$.

Suppose $u^{1}\left(s_{n}\right)=u^{2}\left(s_{n}\right)$. Then, by the continuity of $z,\left(s_{n}, t_{n}\right] \subset \mathscr{B}^{+}$or $\left(s_{n}, t_{n}\right] \subset \mathscr{B}^{-}$. By Lemma 2.1 (i) $z_{1}(t)=z_{2}(t)$ on $\left[s_{n}, t_{n}\right]$, and so $u^{1}\left(t_{n}\right)=u^{2}\left(t_{n}\right)$.

In order to apply the above lemma we need to know when there exist suitable $\alpha_{1}$ and $\alpha_{2}$. This is answered by the following observation.

Lemma 3.8. Let $\rho_{1}, \rho_{2}$ be nondecreasing and continuous on $[0, \tau]$ satisfying $\rho_{1}(0)=$ $\rho_{2}(0)$ and $\rho_{1}(\tau)=\rho_{2}(\tau)$. Let

$$
\mathscr{S}:=\left\{\left(s_{1}, s_{2}\right) \in[0, \tau]^{2}: \rho_{1}\left(s_{1}\right)=\rho_{2}\left(s_{2}\right)\right\} .
$$

Suppose there exist partitions $0=s_{i}^{0}<s_{i}^{1}<\cdots<s_{i}^{N_{i}}=\tau$ such that for each rectangle $Q_{j, k}=\left[s_{1}^{j}, s_{1}^{j+1}\right] \times\left[s_{2}^{k}, s_{2}^{k+1}\right]$ satisfying $Q_{j, k} \cap \mathscr{S} \neq \emptyset$,

(i) $\left[s_{1}^{j}, s_{1}^{j+1}\right] \subset \mathrm{cl}_{+}\left(\mathscr{B}_{\rho_{1}}^{+}\right)$or $\left[s_{2}^{k}, s_{2}^{k+1}\right] \subset \mathrm{cl}_{+}\left(\mathscr{B}_{\rho_{2}}^{+}\right)$where $\mathrm{cl}_{+}$denotes the closure with respect to decreasing sequences, or

(ii) $\left(s_{1}^{j}, s_{1}^{j+1}\right] \cap \mathscr{B}_{\rho_{1}}^{+}=\emptyset$ or $\left(s_{2}^{k}, s_{2}^{k+1}\right] \cap \mathscr{B}_{\rho_{2}}^{+}=\emptyset$.

Then there exists $\alpha_{1}, \alpha_{2} \in \mathscr{A}_{\tau}$ such that $\rho_{1} \circ \alpha_{1}=\rho_{2} \circ \alpha_{2}$.

Proof. Suppose we can find a continuous path $\alpha=\left(\alpha_{1}, \alpha_{2}\right)$ with $\alpha_{1}, \alpha_{2} \in \mathscr{A}_{\tau}$ satisfying $\alpha(t) \in \mathscr{S}$ for all $t \in[0, \tau]$. Then $\rho_{1} \circ \alpha_{1}(t)=\rho_{2} \circ \alpha_{2}(t)$, and we are done. Define the "lower" and "upper" boundary of $Q_{j, k}$ by

$$
\begin{aligned}
& \partial^{-} Q_{j, k}=\left\{s_{1}^{j}\right\} \times\left[s_{2}^{k}, s_{2}^{k+1}\right] \cup\left[s_{1}^{j}, s_{1}^{j+1}\right] \times\left\{s_{2}^{k}\right\}, \\
& \partial^{+} Q_{j, k}=\left\{s_{1}^{j+1}\right\} \times\left[s_{2}^{k}, s_{2}^{k+1}\right] \cup\left[s_{1}^{j}, s_{1}^{j+1}\right] \times\left\{s_{2}^{k+1}\right\} .
\end{aligned}
$$

Claim 1: Let $\tilde{s} \in \partial^{-} Q_{j, k} \cap \mathscr{S}$. Then there exist a (continuous) path $\gamma:[0,1] \rightarrow$ $\mathscr{S} \cap Q_{j, k}$ such that $\gamma(0)=\tilde{s}$ and $\gamma(1) \in \partial^{+} Q_{j, k} \cap \mathscr{S}$. 
Before proving the claim, let us see why the result follows. Let $\mathcal{D}$ be any finite union of squares, i.e., $\mathcal{D}=\cup_{(j, k) \in \mathcal{I}} Q_{j, k}$, where $\mathcal{I} \subset\left\{(j, k): 0 \leq j \leq N_{1}, 0 \leq k \leq N_{2}\right\}$. Then, for such $\mathcal{D}$, let the lower and upper boundary be defined by

$$
\partial^{ \pm} \mathcal{D}=\bigcup_{(j, k) \in \mathcal{I}} \partial^{ \pm} Q_{j, k} \backslash \bigcup_{(j, k) \in \mathcal{I}} \partial^{\mp} Q .
$$

Proceeding by induction on the number of boxes we extend, upon concatenating paths, the result in Claim 1 to any such domain $\mathcal{D}$. But, then Claim 1 is valid for $\mathcal{D}=[0, \tau] \times[0, \tau]$. Consequently, as $(0,0) \in \partial^{-} \mathcal{D} \cap \mathscr{S}$ there exists a continuous path $\gamma$ satisfying $\gamma(0)=(0,0)$ and $\gamma(1) \in \partial^{+} \mathcal{D} \cap \mathscr{S}=([0, \tau] \times\{\tau\} \cup\{\tau\} \times[0, \tau]) \cap \mathscr{S}$. To finish the proof we apply the following observation with $\gamma(1)=s$ and $(\tau, \tau)=\tilde{s}$. Claim 2: Suppose $s, \tilde{s} \in \mathscr{S}$. Then the point

$$
s^{*}:=\left(\max \left\{s_{1}, \tilde{s}_{1}\right\}, \max \left\{s_{2}, \tilde{s}_{2}\right\}\right) \in \mathscr{S} .
$$

Furthermore, the straight line connecting $s$ and $s^{*}$ belongs to $\mathscr{S}$. Similarly for $\tilde{s}$ and $s^{*}$.

It remains to prove Claim 1 and 2.

Proof of Claim 1. Let $g_{i}(\xi):=\min \left\{r \geq \tilde{s}_{i}: \rho_{i}(r) \geq \xi\right\}$ for $i=1,2$. Consider the two paths

$\gamma_{1}(\lambda)=\left(\lambda, g_{2}\left(\rho_{1}(\lambda)\right)\right), \quad \lambda \in\left[\tilde{s}_{1}, s_{1}^{j+1}\right]$, and $\gamma_{2}(\lambda)=\left(g_{1}\left(\rho_{2}(\lambda)\right), \lambda\right), \quad \lambda \in\left[\tilde{s}_{2}, s_{2}^{k+1}\right]$.

Note $\rho_{i}\left(g_{i}(\xi)\right)=\max \left\{\xi, \rho_{i}\left(\tilde{s}_{i}\right)\right\}$, and so, $\gamma_{1}:\left[\tilde{s}_{1}, s_{1}^{j+1}\right] \rightarrow \mathscr{S}$ and $\gamma_{2}:\left[\tilde{s}_{2}, s_{2}^{k+1}\right] \rightarrow$ $\mathscr{S}$. Furthermore, $\gamma_{1}\left(\tilde{s}_{1}\right)=\gamma_{2}\left(\tilde{s}_{2}\right)=\tilde{s}$. Suppose $\left(s_{1}^{j}, s_{1}^{j+1}\right] \cap \mathscr{B}_{\rho_{1}}^{+}=\emptyset$. Then, by Lemma 2.1 (iii) $\rho_{1}$ is constant on $\left[s_{1}^{j}, s_{1}^{j+1}\right]$. Hence, $\gamma_{1}(\lambda)=\left(\lambda, \tilde{s}_{2}\right)$, a straight line connecting $\tilde{s}$ and $\partial^{+} Q_{j, k}$. Similarly, if $\left(s_{2}^{k}, s_{2}^{k+1}\right] \cap \mathscr{B}_{\rho_{2}}^{+}=\emptyset$. To treat case (i), we need the following observation.

(1) $g_{i}$ is left continuous.

(2) $g_{i}$ is right continuous at $\xi$, if for any $\bar{\kappa}>g_{i}(\xi),\left(g_{i}(\xi), \bar{\kappa}\right] \cap \mathscr{B}_{\rho_{i}}^{+} \neq \emptyset$.

Suppose $\left[s_{1}^{j}, s_{1}^{j+1}\right] \subset \operatorname{cl}_{+}\left(\mathscr{B}_{\rho_{1}}^{+}\right)$. It thus follow that $g_{1}$ is continuous as long as $g_{1}\left(\rho_{2}(\lambda)\right) \in\left[s_{1}^{j}, s_{1}^{j+1}\right]$. Consequently, $\gamma_{2}$ is a continuous path connecting $\tilde{s}$ and $\partial^{+} Q_{j, k}$. Similarly, if $\left[s_{2}^{k}, s_{2}^{k+1}\right] \subset \operatorname{cl}_{+}\left(\mathscr{B}_{\rho_{2}}^{+}\right)$.

Let us prove (1) and (2). Let $\xi,\left\{\xi_{n}\right\}_{n \geq 0} \subset\left[\rho_{i}\left(\tilde{s}_{i}\right), \rho_{i}(\tau)\right]$ and suppose $\xi_{n} \uparrow \xi$. Let $g_{i}(\xi)=\kappa$ and $\kappa_{n}=g_{i}\left(\xi_{n}\right)$. We want to show that $\kappa_{n} \uparrow \kappa$. Clearly, $\kappa_{n} \leq \kappa$ for all $n \geq 1$. For any $\varepsilon>0$, there exists $n_{0}(\varepsilon)$ s.t. $\xi-\varepsilon \leq \xi_{n}$ for all $n \geq n_{0}(\varepsilon)$. Consequently, for all $n \geq n_{0}(\varepsilon)$,

$$
\kappa_{n}=\min \left\{r \geq \tilde{s}_{i}: \rho_{i}(r) \geq \xi_{n}\right\} \geq \min \underbrace{\left\{r \geq \tilde{s}_{i}: \rho_{i}(r)+\varepsilon \geq \rho_{i}(\kappa)\right\}}_{V^{\varepsilon}} .
$$

Hence, if $\left\{\kappa_{n}\right\}_{n \geq 0}$ has an accumulation point $\tilde{\kappa}<\kappa$, then $\tilde{\kappa} \in V^{\varepsilon}$ for all $\varepsilon>0$, i.e., $\rho_{i}(\tilde{\kappa}) \geq \rho_{i}(\kappa)$, contradicting that $\kappa=g_{i}(\xi)$. This finishes the proof of (1). Suppose $\xi_{n} \downarrow \xi$. We want to show that $\kappa_{n} \downarrow \kappa$. Again, $\kappa_{n} \geq \kappa$. For any $\varepsilon>0$, there exists $n_{0}(\varepsilon)$ s.t. $\xi+\varepsilon \geq \xi_{n}$ for all $n \geq n_{0}(\varepsilon)$. Hence

$$
\kappa_{n}=\min \left\{r \geq \tilde{s}_{i}: \rho_{i}(r) \geq \xi_{n}\right\} \leq \min \left\{r \geq \tilde{s}_{i}: \rho_{i}(r) \geq \rho_{i}(\kappa)+\varepsilon\right\} .
$$

Suppose $\left\{\kappa_{n}\right\}_{n \geq 0}$ has an accumulation point $\bar{\kappa}>\kappa$. Then

$$
k_{0}:=\inf \left\{r \geq \tilde{s}_{i}: \rho_{i}(r)>\rho_{i}(\kappa)\right\} \geq \bar{\kappa} .
$$


But then $\rho_{i}\left(\kappa_{0}\right)=\rho_{i}(\kappa)$ and so $\left(\kappa, \kappa_{0}\right] \cap \mathscr{B}_{\rho_{i}}^{+}=\emptyset$, a contradiction. This finishes the proof of (2).

Proof of Claim 2. To this end, we might as well assume $s_{1} \leq \tilde{s}_{1}$ and $s_{2} \geq \tilde{s}_{2}$, the other cases beeing either trivial, or analogous. As $\rho_{2}$ is nondecreasing

$$
\rho_{1}\left(\tilde{s}_{1}\right)=\rho_{2}\left(\tilde{s}_{2}\right) \leq \rho_{2}\left(s_{2}\right)=\rho_{1}\left(s_{1}\right),
$$

but then, as $\rho_{1}$ is nondecreasing, $\rho_{1}\left(\tilde{s}_{1}\right)=\rho_{1}\left(s_{1}\right)$. Similarly, $\rho_{2}\left(\tilde{s}_{2}\right)=\rho_{2}\left(s_{2}\right)$. Consequently 3.12 follows. Likewise, it is easily seen that the straight line connecting $s$ and $s^{*}$ belongs to $\mathscr{S}$. Similarly for $\tilde{s}$ and $s^{*}$.

Lemma 3.9. Let $z \in C_{0}([0, \tau])$ and suppose $0 \leq \min \left\{M_{-}, M_{+}\right\}<\infty$. Let $\left\{\tau_{n}\right\}_{n \geq 0}$ be defined in Definition 2.2. Then $\tau_{N}=0$ for some $1 \leq N<\infty$.

Proof. Note that $0 \leq \tau_{n} \leq \tau_{n-1} \leq \tau$ for all $n \geq 1$. And so, $\tau_{n} \downarrow \tau^{*}$ for some $\tau^{*} \in[0, \tau]$. Suppose $\tau_{n}>0$ for all $n \geq 0$. Let $z_{n}:=z\left(\tau_{n}\right), n \geq 0$. Consider the case $\tau_{1} \in \mathscr{B}_{z}^{-}$. By Lemma $2.1(\mathrm{i}), z_{1}=\rho_{z}^{-}\left(\tau_{1}\right)$. Then $z_{2}=\rho_{z}^{+}\left(\tau_{2}\right), z_{3}=\rho_{z}^{-}\left(\tau_{3}\right), \ldots$, and generally

$$
z_{2 n}=\rho_{z}^{+}\left(\tau_{2 n}\right) \quad \text { and } \quad z_{2 n-1}=\rho_{z}^{-}\left(\tau_{2 n-1}\right), \quad n \geq 1 .
$$

Consequently, $z_{2 n} \geq\left(M_{-}\right)^{-1}$ and $z_{2 n-1} \leq-\left(M_{+}\right)^{-1}$ implying that $z\left(\tau_{n}\right) \nrightarrow z\left(\tau^{*}\right)$ as $n \rightarrow \infty$, contradicting the continuity of $z$. The case $\tau_{1} \in \mathscr{B}_{z}^{+}$is analogous. As a result, there exists $0 \leq N<\infty$ suchthat $\tau_{N}=0$.

Lemma 3.10. Suppose $\left(\mathcal{A}_{f}\right.$ holds and $u_{0} \in\left(L^{1} \cap L^{\infty}\right)(\mathbb{R})$ satisfies 2.1). Then there exist a family $\left\{u_{0}^{\varepsilon}\right\}_{\varepsilon>0} \subset\left(L^{1} \cap L^{\infty} \cap B V\right)(\mathbb{R})$ such that $u_{0}^{\varepsilon} \rightarrow u_{0}$ in $L^{1}(\mathbb{R})$ as $\varepsilon \downarrow 0$ and for $y<x$,

$$
-\underbrace{\min \left\{M_{-}, \frac{c}{\varepsilon}\left\|h\left(u_{0}\right)\right\|_{\infty}\right\}}_{M_{-}^{\varepsilon}} \leq \frac{f^{\prime}\left(u_{0}^{\varepsilon}(y)\right)-f^{\prime}\left(u_{0}^{\varepsilon}(x)\right)}{y-x} \leq \underbrace{\min \left\{M_{+}, \frac{c}{\varepsilon}\left\|h\left(u_{0}\right)\right\|_{\infty}\right\}}_{M_{+}^{\varepsilon}},
$$

where $h(u)=\int_{0}^{u} f^{\prime \prime}(z) d z$ and $c>0$ is a constant independent of $f, u_{0}, \varepsilon$.

Proof. Let $J_{\varepsilon}(x)=\varepsilon^{-1} J\left(\varepsilon^{-1} x\right)$, where $J \in C_{c}^{\infty}(\mathbb{R})$ satsifies $\operatorname{supp}(J) \subset(-1,1), J \geq$ 0 , and $\int J=1$. Let $g$ be the inverse of $h$, i.e., $h(g(u))=u$. Define

$$
u_{0}^{\varepsilon}(x):=g\left(\left(h\left(u_{0}\right) \star J_{\varepsilon}\right)(x)\right) .
$$

As $u_{0} \in L^{\infty}(\mathbb{R})$, it follows by $\left(\mathcal{A}_{f}\right)$ that there exist $0<\alpha \leq \beta<\infty$ such that $\alpha \leq h^{\prime}(u) \leq \beta$ for all $|u| \leq\left\|u_{0}\right\|_{\infty}$. Consequently, $\beta^{-1} \leq g^{\prime}(v) \leq \alpha^{-1}$, for all $v \in\left\{h(u):|u| \leq\left\|u_{0}\right\|_{\infty}\right\}$. Also, $g(0)=0$. By Young's inequality for convolutions,

$$
\left\|u_{0}^{\varepsilon}\right\|_{L^{p}(\mathbb{R})} \leq \frac{1}{\alpha}\left\|h\left(u_{0}\right) \star J_{\varepsilon}\right\|_{L^{p}(\mathbb{R})} \leq \frac{1}{\alpha}\left\|h\left(u_{0}\right)\right\|_{L^{p}(\mathbb{R})}\left\|J_{\varepsilon}\right\|_{L^{1}(\mathbb{R})} \leq \frac{\beta}{\alpha}\left\|u_{0}\right\|_{L^{p}(\mathbb{R})},
$$

for any $p \in[1, \infty]$. Similarly,

$$
\begin{aligned}
\left\|u_{0}^{\varepsilon}\right\|_{B V} & \leq \frac{1}{\alpha}\left\|h\left(u_{0}\right) \star J_{\varepsilon}\right\|_{B V} \\
& \leq \frac{1}{\alpha \varepsilon}\left\|h\left(u_{0}\right)\right\|_{L^{1}(\mathbb{R})}\left\|J^{\prime}\right\|_{L^{1}(\mathbb{R})} \\
& \leq \frac{\beta}{\alpha \varepsilon}\left\|u_{0}\right\|_{L^{1}(\mathbb{R})}\left\|J^{\prime}\right\|_{L^{1}(\mathbb{R})} .
\end{aligned}
$$


To prove (3.13) we observe that

$$
\begin{aligned}
\left|f^{\prime}\left(u_{0}^{\varepsilon}(y)\right)-f^{\prime}\left(u_{0}^{\varepsilon}(x)\right)\right| & =\left|h\left(u_{0}^{\varepsilon}(y)\right)-h\left(u_{0}^{\varepsilon}(x)\right)\right| \\
& =\left|\left(h\left(u_{0}\right) \star J_{\varepsilon}\right)(y)-\left(h\left(u_{0}\right) \star J_{\varepsilon}\right)(x)\right| \\
& =\left|\int_{\mathbb{R}} h\left(u_{0}(\xi)\right)\left(J_{\varepsilon}(y-\xi)-J_{\varepsilon}(x-\xi)\right) d \xi\right| \\
& \leq \frac{1}{\varepsilon}\left\|h\left(u_{0}\right)\right\|_{L^{\infty}(\mathbb{R})}\left\|J^{\prime}\right\|_{L^{1}(\mathbb{R})}|y-x| .
\end{aligned}
$$

On the other hand,

$$
\frac{f^{\prime}\left(u_{0}^{\varepsilon}(y)\right)-f^{\prime}\left(u_{0}^{\varepsilon}(x)\right)}{y-x}=\int_{\mathbb{R}} \frac{f^{\prime}\left(u_{0}(y-\xi)\right)-f^{\prime}\left(u_{0}(x-\xi)\right)}{(y-\xi)-(x-\xi)} J_{\varepsilon}(\xi) d \xi .
$$

Combining the two yields (3.13). Finally, for any $1 \leq p \leq \infty$,

$$
\left\|u_{0}^{\varepsilon}-u_{0}\right\|_{L^{p}(\mathbb{R})} \leq \frac{1}{\alpha}\left\|h\left(u_{0}\right) \star J_{\varepsilon}-h\left(u_{0}\right)\right\|_{L^{p}(\mathbb{R})},
$$

and so $u_{0}^{\varepsilon} \rightarrow u_{0}$ in $L^{p}(\mathbb{R})$.

Proof of Theorem 2.4. Assume $u_{0} \in B V(\mathbb{R})$. We construct an approximation of the path $z$ by supplementing the interpolation points $\left\{\left(\tau_{m}, z\left(\tau_{m}\right)\right)\right\}_{m=0}^{N}$, given in Definition 2.2 by points $\left\{\left(t_{m}, z\left(t_{m}\right)\right)\right\}_{m=1}^{\infty}$, This results in a sequence of piecewise linear approximations to $z$, with $z_{0}=\operatorname{Orm}_{\tau, M_{ \pm}}(z)$ and $z_{n}$ the piecewise linear interpolation based on the points $\left\{\left(\tau_{m}, z\left(\tau_{m}\right)\right)\right\}_{m=0}^{N} \cup\left\{\left(t_{m}, z\left(t_{m}\right)\right)\right\}_{m=1}^{n}$. We claim

$$
\begin{aligned}
& \text { For all } n \geq 1, z_{n} \sim z_{0} \text { on }[0, \tau] \text { for all } \\
& u_{0} \in\left(L^{1} \cap L^{\infty} \cap B V\right)(\mathbb{R}) \text { satisfying } 2.1 .
\end{aligned}
$$

Before proving the claim, we verify that Theorem 2.4 follows. As $z$ is uniformly continuous on $[0, \tau]$ we may pick the sequence $\left\{t_{n}\right\}_{n=0}^{\infty}$ such that $z_{n} \rightarrow z$ uniformly on $[0, \tau]$. Let $u^{n}$ be the solution to (1.1) with path $z^{n}$ and initial condition $u_{0}$. By (1.6) it follows that $u^{n}(\tau) \rightarrow u(\tau)$ in $L^{1}(\mathbb{R})$. But by (3.14), $u^{n}(\tau)=u^{0}(\tau)$ for all $n \geq 0$. Hence $u^{0}(\tau)=u(\tau)$ which proves that the equivalence holds for all initial functions $u^{0} \in\left(L^{1} \cap L^{\infty} \cap B V\right)(\mathbb{R})$.

To see that it suffices with $u_{0} \in\left(L^{\infty} \cap L^{1}\right)(\mathbb{R})$ let $\left\{u_{0}^{\varepsilon}\right\}_{\varepsilon>0}$ be the approximation of $u_{0}$ obtained in Lemma 3.10 Let $u^{\varepsilon}$ denote the solution to (1.1) with path $z$ and initial condition $u_{0}^{\varepsilon}$, and $u^{\varepsilon, 0}$ be the solution to 1.1 with path $\operatorname{Orm}_{\tau, M_{ \pm}}(z)$ and initial condition $u_{0}^{\varepsilon}$. Due to the above and (3.13), $u^{\varepsilon}(\tau)=u^{\varepsilon, 0}(\tau)$ for all $\varepsilon>0$. By the continuous dependence estimate (1.6) and the triangle inequality,

$$
\begin{aligned}
\left\|u(\tau)-u^{0}(\tau)\right\|_{L^{1}(\mathbb{R})} & \leq\left\|u(\tau)-u^{\varepsilon}(\tau)\right\|_{L^{1}(\mathbb{R})}+\left\|u^{\varepsilon, 0}(\tau)-u^{0}(\tau)\right\|_{L^{1}(\mathbb{R})} \\
& \leq 2\left\|u_{0}-u_{0}^{\varepsilon}\right\|_{L^{1}(\mathbb{R})},
\end{aligned}
$$

from which Theorem 2.4 follows.

It remains to prove the claim (3.14). Suppose $z_{n-1} \sim z_{0}$, we must show that $z_{n} \sim z_{0}$. For some $1 \leq m \leq N$, we have $\tau_{m} \leq t_{n}<\tau_{m-1}$. Note that $z_{n}\left(\tau_{m}\right)=$ $z_{n-1}\left(\tau_{m}\right)=z\left(\tau_{m}\right)$ and $z_{n}\left(\tau_{m-1}\right)=z_{n-1}\left(\tau_{m-1}\right)=z\left(\tau_{m-1}\right)$, so $z_{n}(t)=z_{n-1}(t)$ for all $t \in\left[0, \tau_{m}\right] \cup\left[\tau_{m-1}, \tau\right]$.

Consider the case $\tau_{m} \in \mathscr{B}_{z}^{-}$. By Lemma 3.8 there exist piecewise linear monotone surjective functions $\alpha_{i}:\left[\tau_{m}, \tau_{m-1}\right] \rightarrow\left[\tau_{m}, \tau_{m-1}\right], i=1,2$, such that $\rho_{z_{n}}^{+} \circ$ $\alpha_{1}=\rho_{z_{n-1}}^{+} \circ \alpha_{2}$ on $\left[\tau_{m}, \tau_{m-1}\right]$. We need to show that $\rho_{z_{n}}^{-} \circ \alpha_{1}=\rho_{z_{n-1}}^{-} \circ \alpha_{2}$ on $\left[\tau_{m}, \tau_{m-1}\right]$. Recall that $\tau_{m}=\max \left\{\mathscr{B}_{z}^{-} \cap\left[0, \tau_{m-1}\right]\right\}$, so that $\left(\tau_{m}, \tau_{m-1}\right] \cap \mathscr{B}_{z}^{-}=\emptyset$. 
By Lemma 2.1 (iii), $\rho_{z}^{-}$is constant on $\left[\tau_{m}, \tau_{m-1}\right]$. Hence, $z(t) \geq \rho_{z}^{-}(t)=\rho_{z}^{-}\left(\tau_{m}\right)=$ $z\left(\tau_{m}\right)$ for $t \in\left(\tau_{m}, \tau_{m-1}\right]$. Accordingly, for any $t \in\left(\tau_{m}, \tau_{m-1}\right]$ and all $n \geq 0$, $z_{n}(t) \geq z\left(\tau_{m}\right)=z_{n}\left(\tau_{m}\right)$, so that

$$
\rho_{z_{n}}^{-}(t)=\min \left\{-\frac{1}{M_{+}}, \min _{0 \leq s \leq t}\left\{z_{n}(s)\right\}\right\}=z\left(\tau_{m}\right), \quad t \in\left[\tau_{m}, \tau_{m-1}\right] .
$$

It follows that $\rho_{z_{n}}^{-} \circ \alpha_{1}=\rho_{z_{n-1}}^{-} \circ \alpha_{2}$ on $\left[\tau_{m}, \tau_{m-1}\right]$. Hence, upon taking

$$
\tilde{\alpha}_{i}(t)= \begin{cases}\alpha_{i}(t) & \text { for } t \in\left[\tau_{m}, \tau_{m-1}\right], \\ t & \text { otherwise, }\end{cases}
$$

we have $\rho_{z_{n}}^{ \pm} \circ \tilde{\alpha}_{1}=\rho_{z_{n-1}}^{ \pm} \circ \tilde{\alpha}_{2}$ on $[0, \tau]$. By Lemma 3.7 it follows that $z_{n} \sim z_{n-1} \sim z_{0}$. The case $\tau_{m} \in \mathscr{B}_{z}^{+}$is treated similarly.

Proof of Theorem 2.5. When $\operatorname{Orm}_{\tau, u_{0}}(z)$ is well-defined, the result is an immediate consequence of Lemma 3.5 and Theorem 2.4. The general case follows by approximation. Let $\left\{u_{0}^{\varepsilon}\right\}_{\varepsilon>0}$ be the approximation of $u_{0}$ obtained in Lemma 3.10 and $u^{\varepsilon}$ be the entropy solution to (1.1) with initial condition $u_{0}^{\varepsilon}$. By the continuous dependence estimate $(1.6)$, it follows that $u^{\varepsilon}(t) \rightarrow u(t)$ in $L^{1}(\mathbb{R})$ as $\varepsilon \downarrow 0$. Let $0 \leq \varphi \in C_{c}^{\infty}\left(\mathbb{R}^{2}\right)$ satisfy $\operatorname{supp}(\varphi) \subset\{(x, y): y>x\}$ and define

$$
\Phi[u, \varphi]:=\iint_{\mathbb{R} \times \mathbb{R}} \frac{f^{\prime}(u(\tilde{y}))-f^{\prime}(u(\tilde{x}))}{\tilde{y}-\tilde{x}} \varphi(\tilde{x}, \tilde{y}) d \tilde{x} d \tilde{y} .
$$

We have that $\Phi\left[u^{\varepsilon}(t), \varphi\right] \rightarrow \Phi[u(t), \varphi]$ as $\varepsilon \rightarrow 0$. By Lemmas 3.5, 3.9, and 3.10, and Theorem 2.4, we conclude that

$$
-\frac{1}{\rho_{z}^{+}(t)-z(t)}\|\varphi\|_{L^{1}(\mathbb{R} \times \mathbb{R})} \leq \Phi\left[u^{\varepsilon}(t), \varphi\right] \leq \frac{1}{z(t)-\rho_{z}^{-}(t)}\|\varphi\|_{L^{1}(\mathbb{R} \times \mathbb{R})},
$$

and so the same holds for $u$. Let $J_{\delta}^{i}(x)=\delta^{-1} J^{i}\left(\delta^{-1} x\right)$, where $J^{i} \in C_{c}^{\infty}(\mathbb{R})$ satisfy $J^{i} \geq 0$ and $\int J^{i}=1, i=1,2$. We also demand that $\operatorname{supp}\left(J^{1}\right) \subset(0,1)$ and $\operatorname{supp}\left(J^{2}\right) \subset(-1,0)$. Fix $y>x$ and let $\varphi(\tilde{x}, \tilde{y})=J_{\delta_{1}}^{1}(x-\tilde{x}) J_{\delta_{2}}^{2}(y-\tilde{y})$. Sending $\delta_{1}, \delta_{2} \downarrow 0$ yields the left (essential) limit at $x$ and the right (essential) limit at $y$ in Theorem 2.5. The general statement follows by modifying the definition of $\varphi$.

Next, we want to apply Theorem 2.4 to prove Theorem 2.8 An important step in this direction is the following observation, which is also of importance for Theorem 2.10

Lemma 3.11. Let $z \in C_{0}([0, \tau])$ be a path for which $\operatorname{Orm}_{\tau, M_{ \pm}}(z)$ is well defined and fix a map $\alpha \in \mathscr{A}_{\tau}$. Denote by $\alpha^{-1}$ the generalized inverse of $\alpha, c f$. (2.9). Then $\mathcal{T}_{z \circ \alpha}^{ \pm}=\left\{\alpha^{-1}(t): t \in \mathcal{T}_{z}^{ \pm}\right\}$, cf. [2.8). It follows that $\mathcal{T}_{z \circ \alpha}=\left\{\alpha^{-1}(t): t \in \mathcal{T}_{z}\right\} \cup\{\tau\}$. Furthermore, $\operatorname{Orm}_{\tau, M_{ \pm}}(z \circ \alpha) \sim \operatorname{Orm}_{\tau, M_{ \pm}}(z)$ for any $u_{0}$ satisfying (2.1).

To prove this statement, we need a more technical result.

Lemma 3.12. Let $z \in C_{0}([0, \tau])$, and fix a nondecreasing surjective map $\alpha$ : $[0, \tau] \rightarrow[0, \tau]$, with generalized inverse $\alpha^{-1}(2.9)$. Then

(i) $\mathscr{B}_{z \circ \alpha}^{ \pm}=\alpha^{-1}\left(\mathscr{B}_{z}^{ \pm}\right) \cap \mathscr{B}_{\alpha}, \mathscr{B}_{\alpha}:=\{t \in[0, \tau]: \min \{s: \alpha(s) \geq \alpha(t)\}=t\}$.

(ii) For a given set $S \subset[0, \tau]$ satisfying $\sup \{S\} \in S$

$$
\max \left\{\alpha^{-1}(S) \cap \mathscr{B}_{\alpha}\right\}=\alpha^{-1}(\max \{S\}) .
$$


(iii) If $\alpha\left(\zeta^{*}\right)=\tau^{*}$ for some $0 \leq \zeta^{*}, \tau^{*} \leq \tau$ then

$$
\left[0, \zeta^{*}\right] \cap \mathscr{B}_{\alpha}=\alpha^{-1}\left(\left[0, \tau^{*}\right]\right) \cap \mathscr{B}_{\alpha} .
$$

Proof. (i). Consider $\mathscr{B}_{z \circ \alpha}^{+}$, with the proof for $\mathscr{B}_{z \circ \alpha}^{-}$being analogous. Since $\alpha$ is nondecreasing,

$$
\rho_{z \circ \alpha}^{+}(t)=\max \left\{\frac{1}{M_{-}}, \max _{0 \leq s \leq \alpha(t)}\{z(s)\}\right\}=\rho_{z}^{+}(\alpha(t)) .
$$

Hence,

$$
\mathscr{B}_{z \circ \alpha}^{+}=\left\{t \in[0, \tau]: \inf \left\{s: \rho_{z}^{+}(\alpha(s)) \geq \rho_{z}^{+}(\alpha(t))\right\}=t\right\} .
$$

Assume $t \in \alpha^{-1}\left(\mathscr{B}_{z}^{+}\right) \cap \mathscr{B}_{\alpha}$ and $\rho_{z}^{+}(\alpha(s)) \geq \rho_{z}^{+}(\alpha(t))$. Then, as $\alpha(t) \in \mathscr{B}_{z}^{+}, \alpha(s) \geq$ $\alpha(t)$. Since $t \in \mathscr{B}_{\alpha}$ it follows that $t \leq s$. Consequently, $t \in \mathscr{B}_{z \circ \alpha}^{+}$. Conversely, if $t \notin \mathscr{B}_{\alpha}$, then there exists $s<t$ such that $\alpha(s)=\alpha(t)$, implying that $t \notin \mathscr{B}_{z \circ \alpha}^{+}$. Similarly, if $\alpha(t) \notin \mathscr{B}_{z}^{+}$there exists $b=\alpha(s)<\alpha(t)$ such that $\rho_{z}^{+}(\alpha(s)) \geq \rho_{z}^{+}(\alpha(t))$. Now, as $\alpha(s)<\alpha(t)$ it follows that $s<t$ and so, $t \notin \mathscr{B}_{z \circ \alpha}^{+}$, thereby proving (i).

(ii). First note that $\alpha^{-1}(\max \{S\}) \in \alpha^{-1}(S) \cap \mathscr{B}_{\alpha}$, which proves $(\geq)$. Conversely, suppose $t \in \alpha^{-1}(S) \cap \mathscr{B}_{\alpha}$. Then

$$
\begin{aligned}
t & =\min \{s \in[0, \tau]: \alpha(s) \geq \alpha(t)\} \\
& \leq \min \{s \in[0, \tau]: \alpha(s) \geq \max \{S\}\} \\
& =\alpha^{-1}(\max \{S\}),
\end{aligned}
$$

which proves $(\leq)$.

(iii). Suppose $0 \leq t \leq \zeta^{*}$. By monotonicity, $t \in \alpha^{-1}\left(\left[0, \tau^{*}\right]\right)$, which proves $(\subset)$. Conversely, assume $t \in \alpha^{-1}\left(\left[0, \tau^{*}\right]\right) \cap \mathscr{B}_{\alpha}$. Then

$$
t=\min \{s: \alpha(s) \geq \alpha(t)\} \leq \min \left\{s: \alpha(s) \geq \tau^{*}\right\}=\alpha^{-1}\left(\tau^{*}\right) \leq \zeta^{*},
$$

implying $(\supset)$.

Proof of Lemma 3.11. Let $\left\{\zeta_{n}\right\}_{n \geq 1}^{M}$ be the interpolation points of $\operatorname{Orm}_{\tau, M_{ \pm}}(z \circ \alpha)$. By definition, $\zeta_{0}=\tau$. Suppose $\tau \notin \mathscr{B}_{z \circ \alpha}^{+} \cup \mathscr{B}_{z \circ \alpha}^{-}$. Applying Lemma 3.12

$$
\begin{aligned}
\zeta_{1} & =\max \left\{\left(\mathscr{B}_{z \circ \alpha}^{+} \cup \mathscr{B}_{z \circ \alpha}^{-}\right) \cap[0, \tau]\right\} \\
& =\max \left\{\alpha^{-1}\left(\left(\mathscr{B}_{z}^{+} \cup \mathscr{B}_{z}^{-}\right) \cap[0, \tau]\right) \cap \mathscr{B}_{\alpha}\right\} \\
& =\alpha^{-1}\left(\tau_{1}\right) .
\end{aligned}
$$

Suppose $\zeta_{n}=\alpha^{-1}\left(\tau_{n}\right)$. If $\tau_{n} \in \mathscr{B}_{z}^{ \pm}$, then by Lemma 3.12 (i), $\zeta_{n} \in \mathscr{B}_{z \circ \alpha}^{ \pm}$. By (i), (ii), and (iii),

$$
\zeta_{n+1}=\max \left\{\mathscr{B}_{z \circ \alpha}^{ \pm} \cap\left[0, \zeta_{n}\right]\right\}=\max \left\{\alpha^{-1}\left(\mathscr{B}_{z}^{ \pm} \cap\left[0, \tau_{n}\right]\right) \cap \mathscr{B}_{\alpha}\right\}=\alpha^{-1}\left(\tau_{n+1}\right) .
$$

Consequently $\zeta_{n}=\alpha^{-1}\left(\tau_{n}\right)$ for all $1 \leq n \leq N$. As $\zeta_{N}=0$ and $\alpha^{-1}(0)=0$ it follows that $\zeta_{N}=0$, and so $M=N$. Consider the last statement. Let us denote by $\mathscr{L}\left(\left\{\left(t_{n}, z_{n}\right)_{n=0}^{N}\right\}\right)$ the piecewise linear interpolation of the points $\left\{\left(t_{n}, z_{n}\right)_{n=0}^{N}\right\}$. Set $\psi=\mathscr{L}\left(\left\{\tau_{n}, \zeta_{n}\right\}_{n=0}^{N}\right)$. By the above, $\alpha\left(\zeta_{n}\right)=\tau_{n}$ and so

$$
\begin{aligned}
\operatorname{Orm}_{\tau, u_{0}}(z \circ \alpha) \circ \psi=\mathscr{L}\left(\left\{\zeta_{n}, z \circ \alpha\left(\zeta_{n}\right)\right\}\right) \circ \psi & =\mathscr{L}\left(\left\{\tau_{n}, z \circ \alpha\left(\zeta_{n}\right)\right\}\right) \\
& =\mathscr{L}\left(\left\{\tau_{n}, z\left(\tau_{n}\right)\right\}\right)=\operatorname{Orm}_{\tau, u_{0}}(z) .
\end{aligned}
$$

As a consequence, cf. Corollary 3.6 it follows that $\operatorname{Orm}_{\tau, u_{0}}(z \circ \alpha) \sim \operatorname{Orm}_{\tau, u_{0}}(z)$. 
Proof of Theorem 2.8. Let $\left\{u_{0}^{\varepsilon}\right\}_{\varepsilon>0}$ be the approximation of $u_{0}$ from Lemma 3.10 . Let us show that $z_{1} \sim z_{2}$ on $[0, \tau]$ with initial condition $u_{0}^{\varepsilon}$. Note first that $\rho_{z_{1}, \varepsilon}^{ \pm} \circ \alpha_{1}=\rho_{z_{2}, \varepsilon}^{ \pm} \circ \alpha_{2}$, where $\rho_{z_{1}, \varepsilon}^{ \pm}, \rho_{z_{2}, \varepsilon}^{ \pm}$are defined with respect to $M_{-}^{\varepsilon}, M_{+}^{\varepsilon}$, cf. Lemma 3.10. By Theorem 2.4 it suffices to prove $\operatorname{Orm}_{\tau, u_{0}^{\varepsilon}}\left(z_{1}\right) \sim \operatorname{Orm}_{\tau, u_{0}^{\varepsilon}}\left(z_{2}\right)$. However, by Lemma 3.11, we have

$$
\operatorname{Orm}_{\tau, u_{0}^{\varepsilon}}\left(z_{1}\right) \sim \operatorname{Orm}_{\tau, u_{0}^{\varepsilon}}\left(z_{1} \circ \alpha_{1}\right)=\operatorname{Orm}_{\tau, u_{0}^{\varepsilon}}\left(z_{2} \circ \alpha_{2}\right) \sim \operatorname{Orm}_{\tau, u_{0}^{\varepsilon}}\left(z_{2}\right) .
$$

Let $u_{i}^{\varepsilon}$ be the entropy solution to (1.1) on $[0, \tau]$ with initial condition $u_{0}^{\varepsilon}$ and path $z_{i}$, $i=1,2$. By the above, $u_{1}^{\varepsilon}(\tau)=u_{2}^{\varepsilon}(\tau)$. Applying (1.6) and the triangle inequality it follows that

$$
\begin{aligned}
\left\|u_{1}(\tau)-u_{2}(\tau)\right\|_{L^{1}(\mathbb{R})} & \leq\left\|u_{1}(\tau)-u_{1}^{\varepsilon}(\tau)\right\|_{L^{1}(\mathbb{R})}+\left\|u_{2}^{\varepsilon}(\tau)-u_{2}(\tau)\right\|_{L^{1}(\mathbb{R})} \\
& \leq 2\left\|u_{0}^{\varepsilon}(\tau)-u_{0}(\tau)\right\|_{L^{1}(\mathbb{R})} \rightarrow 0,
\end{aligned}
$$

as $\varepsilon \downarrow 0$. Hence, $u_{1}(\tau)=u_{2}(\tau)$.

Next, we turn to the proof of Theorem 2.10 We first make some observations regarding the properties of the map $\Phi$ defined in Theorem 2.10 .

Lemma 3.13. Let $\Phi$ be defined in Theorem 2.10 and $z_{1}, z_{2} \in C_{0}([0, \tau])$ be paths such that $\operatorname{Orm}_{\tau, M_{ \pm}^{i}}\left(z_{i}\right), i=1,2$, are well defined. Then the following statements are true:

(i) The map $\Phi$ is symmetric in the sense that

$$
\Phi\left[z_{1}, z_{2}\right](\alpha, \beta)=\Phi\left[z_{2}, z_{1}\right](\beta, \alpha), \quad \alpha, \beta \in \mathscr{A}_{\tau} .
$$

(ii) The map $\alpha \mapsto \Phi\left[z_{1}, z_{2}\right](\alpha, \beta)$, defined for $\alpha, \beta \in \mathscr{A}_{\tau}$, is continuous with respect to "uniform convergence from above", i.e., if $\left\{\alpha_{j}\right\}_{j \geq 0} \subset \mathscr{A}_{\tau}$ satisfies $\alpha_{j} \downarrow \alpha$ uniformly on $[0, \tau]$ as $j \rightarrow \infty$, then

$$
\Phi\left[z_{1}, z_{2}\right]\left(\alpha_{j}, \beta\right) \rightarrow \Phi\left[z_{1}, z_{2}\right](\alpha, \beta) \text { as } j \rightarrow \infty .
$$

(iii) Suppose $\tilde{z}_{1} \in C_{0}([0, \tau])$ satisfies $\rho_{z_{1}}^{ \pm} \circ \alpha_{1}=\rho_{\tilde{z}_{1}}^{ \pm} \circ \beta_{1}$ for some $\alpha_{1}, \beta_{1} \in \mathscr{A}_{\tau}$ and $\tilde{z}_{1}(\tau)=z_{1}(\tau)$. Then

$$
\Phi\left[z_{1}, z_{2}\right]\left(\alpha_{1} \circ \zeta, \beta\right)=\Phi\left[\tilde{z}_{1}, z_{2}\right]\left(\beta_{1} \circ \zeta, \beta\right), \quad \zeta, \beta \in \mathscr{A}_{\tau} .
$$

Proof. Set

$$
\mathscr{S}^{ \pm}\left[z_{1}, z_{2}\right](\alpha, \beta):=\left\{\left|\rho_{z_{1}}^{ \pm}(\alpha(t))-\rho_{z_{2}}^{ \pm}(\beta(t))\right|: t \in \mathcal{T}_{z_{1} \circ \alpha}^{ \pm} \cup \mathcal{T}_{z_{2} \circ \beta}^{ \pm}\right\},
$$

so that

$$
\Phi\left[z_{1}, z_{2}\right](\alpha, \beta)=\max _{ \pm}\left\{\mathscr{S}^{ \pm}\left[z_{1}, z_{2}\right](\alpha, \beta) \cup\left\{\left|z_{1}(\tau)-z_{2}(\tau)\right|\right\}\right\} .
$$

(i) The statement is a trivial consequence of the definition.

(ii) By Lemma 3.11 and the fact that $\alpha_{j}\left(\alpha_{j}^{-1}(t)\right)=t$,

$$
\begin{aligned}
\mathscr{S}^{ \pm}\left[z_{1}, z_{2}\right]\left(\alpha_{j}, \beta\right)= & \left\{\left|\rho_{z_{1}}^{ \pm}(t)-\rho_{z_{2}}^{ \pm}\left(\beta\left(\alpha_{j}^{-1}(t)\right)\right)\right|: t \in \mathcal{T}_{z_{1}}^{ \pm}\right\} \\
& \cup\left\{\left|\rho_{z_{1}}^{ \pm}\left(\alpha_{j}(t)\right)-\rho_{z_{2}}^{ \pm}(\beta(t))\right|: t \in \mathcal{T}_{z_{2} \circ \beta}^{ \pm}\right\} .
\end{aligned}
$$

By assumption, as $j \rightarrow \infty, \alpha_{j} \downarrow \alpha$ uniformly on $[0, \tau]$. It remains to verify that $\alpha_{j}^{-1}(t) \uparrow \alpha^{-1}(t)$ for any $t \in[0, \tau]$. Let $\delta>0$. By assumption there exists $j_{0}(\delta) \geq 0$ such that

$$
\alpha(s) \leq \alpha_{j}(s) \leq \alpha(s)+\delta \text { for all } j \geq j_{0}(\delta) \text { and } s \in[0, \tau]
$$


Hence, for $j \geq j_{0}(\delta)$,

$$
\begin{aligned}
\alpha^{-1}(t) & =\min \{0 \leq s \leq \tau: \alpha(s) \geq t\} \\
& \geq \min \left\{0 \leq s \leq \tau: \alpha_{j}(s) \geq t\right\}=\alpha_{j}^{-1}(t) \\
& \geq \min \{0 \leq s \leq \tau: \alpha(s) \geq t-\delta\}=\alpha^{-1}(t-\delta) .
\end{aligned}
$$

The result now follows since $\alpha^{-1}$ is left-continuous.

(iii) Observe that

$$
\begin{aligned}
\mathscr{S}^{ \pm}\left[z_{1}, z_{2}\right]\left(\alpha_{1} \circ \zeta, \beta\right): & =\left\{\left|\rho_{z_{1}}^{ \pm}\left(\alpha_{1} \circ \zeta(t)\right)-\rho_{z_{2}}^{ \pm}(\beta(t))\right|: t \in \mathcal{T}_{z_{1} \circ \alpha_{1} \circ \zeta}^{ \pm} \cup \mathcal{T}_{z_{2} \circ \beta}^{ \pm}\right\} \\
& =\left\{\mid \rho_{\tilde{z}_{1}}^{ \pm}\left(\beta_{1} \circ \zeta(t)\right)-\rho_{z_{2}}^{ \pm}\left(\beta(t) \mid: t \in \mathcal{T}_{z_{1} \circ \alpha_{1} \circ \zeta}^{ \pm} \cup \mathcal{T}_{z_{2} \circ \beta}^{ \pm}\right\} .\right.
\end{aligned}
$$

By assumption, $\mathcal{T}_{z_{1} \circ \alpha_{1}}^{ \pm}=\mathcal{T}_{\tilde{z}_{1} \circ \beta_{1}}^{ \pm}$and so, by Lemma 3.11. $\mathcal{T}_{z_{1} \circ \alpha_{1} \circ \zeta}^{ \pm}=\mathcal{T}_{\tilde{z}_{1} \circ \beta_{1} \circ \zeta}^{ \pm}$. It follows that

$$
\mathscr{S}^{ \pm}\left[z_{1}, z_{2}\right]\left(\alpha_{1} \circ \zeta, \beta\right)=\mathscr{S}^{ \pm}\left[\tilde{z}_{1}, z_{2}\right]\left(\beta_{1} \circ \zeta, \beta\right) .
$$

The result now follows as $\tilde{z}_{1}(\tau)=z_{1}(\tau)$.

Lemma 3.14. Let $z \in C_{0}([0, \tau])$ and suppose $\operatorname{Orm}_{\tau, M_{ \pm}}(z)$ is well defined. Let $\mathscr{S}^{ \pm}$ be disjoint finite subsets of $(0, \tau)$ such that $\mathcal{T}_{z}^{ \pm} \subset \mathscr{S}^{ \pm}$. Let $\Gamma^{ \pm}: \mathscr{S}^{ \pm} \rightarrow \mathbb{R}^{ \pm}$satisfy

$$
\begin{cases}\Gamma^{ \pm}(t)=\rho_{z}^{ \pm}(t), & t \in \mathcal{T}_{z}^{ \pm}, \\ \Gamma^{+}(t) \leq \rho_{z}^{+}(t), & t \in \mathscr{S}^{+}, \\ \Gamma^{-}(t) \geq \rho_{z}^{-}(t), & t \in \mathscr{S}^{-} .\end{cases}
$$

\section{Define}

$$
\mathcal{I}=\left\{\left(t, \Gamma^{+}(t)\right), t \in \mathscr{S}^{+}\right\} \cup\left\{\left(t, \Gamma^{-}(t)\right), t \in \mathscr{S}^{-}\right\} \cup\{(0,0),(\tau, z(\tau))\} .
$$

Let $\tilde{z}$ be the piecewise linear interpolation of $\mathcal{I}$, i.e., $\tilde{z}=\mathcal{L}(\mathcal{I})$. Then $\tilde{z} \sim z$ on $[0, \tau]$ for any initial value $u_{0}$ satisfying 2.1.

Proof. Let $\left\{\tau_{n}\right\}_{n>0}^{N}=\mathcal{T}_{z}$ and $\left\{\tilde{\tau}_{n}\right\}_{n>0}^{\tilde{N}}=\mathcal{T}_{\tilde{z}}$. We want to show that $\mathcal{T}_{\tilde{z}}=\mathcal{T}_{z}$. The result then follows by Theorem 2.4 as $\operatorname{Orm}_{\tau, M_{ \pm}}(z)=\operatorname{Orm}_{\tau, M_{ \pm}}(\tilde{z})$. First observe, for any $t \in \mathscr{S}^{+}$,

$$
\begin{aligned}
\rho_{\tilde{z}}^{+}(t) & =\max \left\{\frac{1}{M_{-}}, \max _{0 \leq s \leq t}\{\tilde{z}(s)\}\right\} \\
& =\max \left\{\frac{1}{M_{-}}, \max \left\{\Gamma^{+}(s): s \in[0, t] \cap \mathscr{S}^{+}\right\}\right\} \leq \rho_{z}^{+}(t),
\end{aligned}
$$

with equality whenever $t \in \mathcal{T}_{z}^{+}$. Recall that $\tau_{1}:=\max \left\{\mathscr{B}_{z}^{+} \cup \mathscr{B}_{z}^{-} \cap[0, \tau]\right\}$, we want to show that $\tilde{\tau}_{1}=\tau_{1}$. As $\left(\tau_{1}, \tau\right] \cap \mathscr{B}_{z}^{+}=\emptyset$ it follows by Lemma 2.1 (iii) that $\rho_{z}^{+}$is constant on $\left[\tau_{1}, \tau\right]$. Consequently, by the above, the same holds for $\rho_{\tilde{z}}^{+}$. Similarly, $\rho_{\tilde{z}}^{-}$ is constant on $\left[\tau_{1}, \tau\right]$. Suppose $\tau_{1} \in \mathscr{B}_{z}^{+}$and let $t=\max \left\{\{0\} \cup \mathscr{S}^{+} \cup \mathscr{S}^{-} \cap\left[0, \tau_{1}\right)\right\}$. As $\tau_{1} \in \mathscr{B}_{z}^{+}$we have $\rho_{z}^{+}(t)<\rho_{z}^{+}\left(\tau_{1}\right)$. By the above inequality,

$$
\partial_{-} \rho_{\tilde{z}}^{+}\left(\tau_{1}\right)=\frac{\rho_{\tilde{z}}^{+}\left(\tau_{1}\right)-\rho_{\tilde{z}}^{+}(t)}{\tau_{1}-t} \geq \frac{\rho_{z}^{+}\left(\tau_{1}\right)-\rho_{z}^{+}(t)}{\tau_{1}-t}>0 .
$$

Hence, by Lemma 2.1 (iv), $\tau_{1} \in \mathscr{B}_{\tilde{z}}^{+}$. It does follow that $\tilde{\tau}_{1} \geq \tau_{1}$ and so $\tilde{\tau}_{1}=\tau_{1}$. Similar argument holds if $\tau_{1} \in \mathscr{B}_{z}^{-}$. The proof now follows by induction, each step being similar to the one above.

The next result contains the main ideas needed in the proof of Theorem 2.10 . 
Lemma 3.15. Fix two paths $z_{1}, z_{2} \in C_{0}([0, \tau])$ and let $\Phi$ be the function defined in Theorem 2.10, Then

(i) $\Phi\left[z_{1}, z_{2}\right](\iota, \iota) \leq\left\|z_{1}-z_{2}\right\|_{\infty}$.

(ii) Suppose $\alpha_{1}, \alpha_{2} \in \mathscr{A}_{\tau}$ satisfy $\mathcal{T}_{z_{1} \circ \alpha_{1}}^{+} \cap \mathcal{T}_{z_{2} \circ \alpha_{2}}^{-}=\emptyset, \mathcal{T}_{z_{1} \circ \alpha}^{-} \cap \mathcal{T}_{z_{2} \circ \alpha_{2}}^{+}=\emptyset$, and $\alpha_{i}^{-1}(\tau)<\tau$ for $i=1,2$. Then there exists $\tilde{z}_{i}$ with $z_{i} \stackrel{\tau, M_{ \pm}^{i}}{\sim} \tilde{z}_{i}, i=1,2$, and

$$
\Phi\left[z_{1}, z_{2}\right]\left(\alpha_{1}, \alpha_{2}\right)=\left\|\tilde{z}_{1}-\tilde{z}_{2}\right\|_{\infty} .
$$

Proof. For $a, b \in \mathbb{R}$, let $a \wedge b:=\min \{a, b\}$ and $a \vee b:=\max \{a, b\}$. Consider (i). Pick $t \in\left(\kappa^{+}, \tau\right]$ and suppose $\rho_{z_{1}}^{+}(t)>\rho_{z_{2}}^{+}(t)$. As $t>\kappa^{+}$,

$$
\rho_{z_{1}}^{+}(t)=\rho_{z_{1}}^{+}(t) \vee \rho_{z_{2}}^{+}(t)>\frac{1}{M_{-}^{1}} \vee \frac{1}{M_{-}^{2}} \geq \rho_{z_{1}}^{+}(0) .
$$

By Lemma 2.1 (iii), $(0, t] \cap \mathscr{B}_{z_{1}}^{+} \neq \emptyset$. Hence, $\xi:=\max \left\{[0, t] \cap \mathscr{B}_{z_{1}}^{+}\right\}>0$. As $(\xi, t] \cap \mathscr{B}_{z_{1}}^{+}=\emptyset$, it follows by Lemma 2.1 (i) and (iii) that $z_{1}(\xi)=\rho_{z_{1}}^{+}(\xi)=\rho_{z_{1}}^{+}(t)$. Hence,

$$
\rho_{z_{1}}^{+}(t)-\rho_{z_{2}}^{+}(t)=z_{1}(\xi)-z_{2}(\xi)+\underbrace{\left(z_{2}(\xi)-\rho_{z_{2}}^{+}(\xi)\right)}_{\leq 0}+\underbrace{\left(\rho_{z_{2}}^{+}(\xi)-\rho_{z_{2}}^{+}(t)\right)}_{\leq 0} .
$$

A similar argument holds for $\rho_{z_{1}}^{+}(t)<\rho_{z_{2}}^{+}(t)$. Consequently,

$$
\left|\rho_{z_{1}}^{+}(t)-\rho_{z_{2}}^{+}(t)\right| \leq\left\|z_{1}-z_{2}\right\|_{\infty}, \quad \text { for any } t \in\left(\kappa^{+}, \tau\right] .
$$

Similarly,

$$
\left|\rho_{z_{1}}^{-}(t)-\rho_{z_{2}}^{-}(t)\right| \leq\left\|z_{1}-z_{2}\right\|_{\infty}, \text { for any } t \in\left(\kappa^{-}, \tau\right],
$$

which finishes the proof of (i).

Consider (ii). Let $\zeta_{i}=z_{i} \circ \alpha_{i}$ for $i=1,2$. We want to apply Lemma 3.14 to construct suitable $\tilde{z}_{1}, \tilde{z}_{2}$. Let $\mathscr{S}^{ \pm}:=\mathcal{T}_{\zeta_{1}}^{ \pm} \cup \mathcal{T}_{\zeta_{2}}^{ \pm}$. By assumption and Lemma 2.1 (i),

$$
\mathscr{S}^{+} \cap \mathscr{S}^{-}=\left(\mathcal{T}_{\zeta_{1}}^{+} \cup \mathcal{T}_{\zeta_{2}}^{+}\right) \cap\left(\mathcal{T}_{\zeta_{1}}^{-} \cup \mathcal{T}_{\zeta_{2}}^{-}\right)=\left(\mathcal{T}_{\zeta_{1}}^{+} \cap \mathcal{T}_{\zeta_{2}}^{-}\right) \cup\left(\mathcal{T}_{\zeta_{1}}^{-} \cap \mathcal{T}_{\zeta_{2}}^{+}\right)=\emptyset .
$$

By Lemma 3.12 (i), $\mathscr{B}_{z_{i} \circ \alpha_{i}}^{ \pm}=\alpha_{i}^{-1}\left(\mathscr{B}_{z_{i}}^{ \pm}\right) \cap \mathscr{B}_{\alpha_{i}}$. As $\alpha_{i}^{-1}(\tau)<\tau$ so that $\tau \notin \mathscr{B}_{\alpha_{i}}$ it follows that $\tau \notin \mathcal{T}_{\zeta_{i}}^{ \pm}$for $i=1,2$. Let

$\Gamma_{j}^{+}(t)=\left\{\begin{array}{ll}\rho_{\zeta_{1}}^{+}(t) \wedge \rho_{\zeta_{2}}^{+}(t), & t \in\left[0, \kappa^{+}\right], \\ \rho_{\zeta_{j}}^{+}(t), & t \in\left(\kappa^{+}, \tau\right],\end{array} \quad \Gamma_{j}^{-}(t)= \begin{cases}\rho_{\zeta_{1}}^{-}(t) \vee \rho_{\zeta_{2}}^{-}(t), & t \in\left[0, \kappa^{-}\right], \\ \rho_{\zeta_{j}}^{+}(t), & t \in\left(\kappa^{-}, \tau\right] .\end{cases}\right.$

In order to apply Lemma 3.14, it remains to verify that $\Gamma_{i}^{ \pm}(t)=\rho_{\zeta_{i}}^{ \pm}(t)$ for any $t \in \mathcal{T}_{\zeta_{i}}^{ \pm}, i=1,2$. We consider $\Gamma_{1}^{+}$, the proof for the others are similar. It is obviously true, for any $t \in \mathcal{T}_{\zeta_{1}}^{+} \cap\left(\kappa^{+}, \tau\right]$. Suppose $\frac{1}{M_{-}^{1}} \geq \frac{1}{M_{-}^{2}}$. Then $\rho_{\zeta_{1}}^{+}\left(\kappa^{+}\right)=\frac{1}{M_{-}^{1}}=\rho_{\zeta_{1}}^{+}(0)$ and so $\left(0, \kappa^{+}\right] \cap \mathscr{B}_{\zeta_{1}}^{+}=\emptyset$. Hence $\mathcal{T}_{\zeta_{1}}^{+} \cap\left[0, \kappa^{+}\right]=\emptyset$, and we are done. Suppose $\frac{1}{M_{-}^{1}} \leq \frac{1}{M_{-}^{2}}$. Then, for any $t \in\left[0, \kappa^{+}\right]$,

$$
\rho_{\zeta_{2}}^{+}(t)=\rho_{\zeta_{2}}\left(\kappa^{+}\right) \geq \rho_{\zeta_{1}}^{+}\left(\kappa^{+}\right) \geq \rho_{\zeta_{1}}^{+}(t),
$$

and so $\rho_{\zeta_{1}}^{+}(t) \wedge \rho_{\zeta_{2}}^{+}(t)=\rho_{\zeta_{1}}^{+}(t)$, and we are done. It remains to verify that

$$
\left\|\tilde{z}_{1}-\tilde{z}_{2}\right\|_{\infty}=\max \left\{\left|\tilde{z}_{1}(t)-\tilde{z}_{2}(t)\right|: t \in \mathcal{T}_{\zeta_{1}} \cup \mathcal{T}_{\zeta_{2}}\right\}=\Phi\left[z_{1}, z_{2}\right]\left(\alpha_{1}, \alpha_{2}\right) .
$$


The first equality follows since $\tilde{z}_{1}, \tilde{z}_{2}$ are piecewise linear, while the second equality follows as

$$
\begin{aligned}
\max \left\{\left|\tilde{z}_{1}(t)-\tilde{z}_{2}(t)\right|: t \in \mathcal{T}_{\zeta_{1}} \cup \mathcal{T}_{\zeta_{2}}\right\}= & \max \left\{\left|\Gamma_{1}^{+}(t)-\Gamma_{2}^{+}(t)\right|: t \in \mathscr{S}^{+} \cap\left(\kappa^{+}, \tau\right]\right\} \\
& \left.\vee \max \left\{\left|\Gamma_{1}^{-}(t)-\Gamma_{2}^{-}(t)\right|: t \in \mathscr{S}^{-} \cap\left(\kappa^{-}, \tau\right]\right)\right\} \\
& \vee\left|z_{1}(\tau)-z_{2}(\tau)\right| .
\end{aligned}
$$

This finishes the proof of (ii).

Before we turn to the proof of Theorem 2.10, we need

Lemma 3.16. Let $\alpha \in \mathscr{A}_{\tau}$ satisfy $\alpha(t)>0$ for all $t \in(0, \tau]$. Then, for any $\xi \in \mathscr{A}_{\tau}$, there exists $\zeta^{\varepsilon} \in \mathscr{A}_{\tau}$ such that $\alpha \circ \zeta^{\varepsilon} \downarrow \xi$ as $\varepsilon \downarrow 0$ with respect to uniform convergence.

Proof. Let $\breve{\alpha}$ be the lower convex envelope of $\alpha$ :

$$
\breve{\alpha}(t)=\sup \{\beta(t): \beta \leq \alpha \text { and } \beta \text { is convex on }[0, \tau]\} \text {. }
$$

Then $\breve{\alpha}$ is strictly increasing. Let $\xi \in \mathscr{A}$ and define

$$
\alpha^{\varepsilon}=(1-\varepsilon) \alpha+\varepsilon \breve{\alpha}, \quad \xi=\alpha^{\varepsilon} \circ \zeta^{\varepsilon}, \quad \xi^{\varepsilon}=\alpha \circ \zeta^{\varepsilon} .
$$

Then $\xi^{\varepsilon}-\xi=\varepsilon(\alpha-\breve{\alpha}) \circ \zeta^{\varepsilon}$ so that $\xi \leq \xi^{\varepsilon} \leq \xi+2 \varepsilon \tau$.

Proof of Theorem 2.10. The theorem is a direct consequence of claims 1 and 2.

Claim 1: We have

$$
\left\|\left[z_{1}\right]-\left[z_{2}\right]\right\|_{\infty}=\inf \left\{\Phi\left[\tilde{z}_{1}, \tilde{z}_{2}\right](\iota, \iota): \tilde{z}_{1} \sim z_{1}, \tilde{z}_{2} \sim z_{2}\right\} .
$$

By Lemma 3.15 (i),

$$
\begin{aligned}
& \left\|\left[z_{1}\right]_{\tau, M_{ \pm}^{1}}-\left[z_{2}\right]_{\tau, M_{ \pm}^{2}}\right\|_{\infty}=\inf \left\{\left\|\tilde{z}_{1}-\tilde{z}_{2}\right\|_{\infty}: \tilde{z}_{1} \stackrel{\tau, M_{ \pm}^{1}}{\sim} z_{1}, \tilde{z}_{2} \stackrel{\tau, M_{ \pm}^{2}}{\sim} z_{2}\right\} \\
& \geq \underbrace{\inf \left\{\Phi\left[\tilde{z}_{1}, \tilde{z}_{2}\right](\iota, \iota): \tilde{z}_{1} \stackrel{\tau, M_{ \pm}^{1}}{\sim} z_{1}, \tilde{z}_{2} \stackrel{\tau, M_{ \pm}^{2}}{\sim} z_{2}\right\}}_{I} .
\end{aligned}
$$

Let $\varepsilon>0$. Pick $\tilde{z}_{1}, \tilde{z}_{2} \in C_{0}([0, \tau])$ such that $\tilde{z}_{1} \stackrel{\tau, M_{ \pm}^{1}}{\sim} z_{1}, \tilde{z}_{2} \stackrel{\tau, M_{ \pm}^{2}}{\sim} z_{2}$ satisfy

$$
I+\varepsilon>\Phi\left[\tilde{z}_{1}, \tilde{z}_{2}\right](\iota, \iota)
$$

For any $\delta>0$, define

$$
\alpha_{i}^{\delta}(t)= \begin{cases}(1+\delta) t, & t \in[0, \tau /(1+\delta)], \\ \tau, & t \in[\tau /(1+\delta), \tau],\end{cases}
$$

for $i=1,2$. Then, for any $\delta_{0}, \eta_{0}>0$, there exist $0<\delta<\delta_{0}, 0<\eta<\eta_{0}$ such that

$$
\mathcal{T}_{\tilde{z}_{1} \circ \alpha_{1}^{\delta}}^{+} \cap \mathcal{T}_{\tilde{z}_{2} \circ \alpha_{2}^{\eta}}^{-}=\emptyset, \quad \mathcal{T}_{\tilde{z}_{1} \circ \alpha_{1}^{\delta}}^{-} \cap \mathcal{T}_{\tilde{z}_{2} \circ \alpha_{2}^{\eta}}^{+}=\emptyset,
$$

and $\alpha_{i}^{-1}(\tau)<\tau$ for $i=1,2$. Consequently, by Lemma 3.13 (ii) and 3.15 (ii) there exist $\delta, \eta>0$ and $\tilde{z}_{1}, \tilde{z}_{2}$ with $\tilde{z}_{1} \stackrel{\tau, M_{ \pm}^{1}}{\sim} z_{1}, \tilde{z}_{2} \stackrel{\tau, M_{ \pm}^{2}}{\sim} z_{2}$ such that

$$
\left|\Phi\left[\tilde{z}_{1}, \tilde{z}_{2}\right]\left(\alpha_{1}^{\delta}, \alpha_{2}^{\eta}\right)-\Phi\left[\tilde{z}_{1}, \tilde{z}_{2}\right](\iota, \iota)\right| \leq \varepsilon, \quad \Phi\left[\tilde{z}_{1}, \tilde{z}_{2}\right]\left(\alpha_{1}^{\delta}, \alpha_{2}^{\eta}\right)=\left\|\tilde{z}_{1}-\tilde{z}_{1}\right\|_{\infty} .
$$

As a result,

$$
I+2 \varepsilon>\Phi\left[\tilde{z}_{1}, \tilde{z}_{2}\right]\left(\alpha_{1}^{\delta}, \alpha_{2}^{\eta}\right)=\left\|\tilde{z}_{1}^{\alpha}-\tilde{z}_{1}^{\alpha}\right\|_{\infty} \geq\left\|\left[z_{1}\right]_{\tau, M_{ \pm}^{1}}-\left[z_{2}\right]_{\tau, M_{ \pm}^{2}}\right\|_{\infty},
$$


finishing the proof of Claim 1.

Claim 2: Define

$$
\begin{aligned}
& \mathscr{I}_{1}\left[z_{1}, z_{2}\right]:=\left\{\Phi\left[z_{1}, z_{2}\right](\alpha, \beta): \alpha, \beta \in \mathscr{A}\right\}, \\
& \mathscr{I}_{2}\left[z_{1}, z_{2}\right]:=\left\{\Phi\left[\tilde{z}_{1}, \tilde{z}_{2}\right](\iota, \iota): \tilde{z}_{1} \stackrel{\tau, M_{ \pm}^{1}}{\sim} z_{1}, \tilde{z}_{2} \stackrel{\tau, M_{ \pm}^{2}}{\sim} z_{2}\right\} .
\end{aligned}
$$

Then $\operatorname{cl}\left(\mathscr{I}_{1}\left[z_{1}, z_{2}\right]\right)=\mathscr{I}_{2}\left[z_{1}, z_{2}\right]$. Therefore, inf $\mathscr{I}_{1}=\inf \mathscr{I}_{2}$.

We first prove that $\mathscr{I}_{1}\left[z_{1}, z_{2}\right] \subset \mathscr{I}_{2}\left[z_{1}, z_{2}\right]$. For any $s \in \mathscr{I}_{1}$ there exists $\alpha_{1}, \alpha_{2} \in$ $\mathscr{A}$ such that $s=\Phi\left[z_{1}, z_{2}\right]\left(\alpha_{1}, \alpha_{2}\right)$. Let $\tilde{z}_{i}=z_{i} \circ \alpha_{i}, i=1,2$. It follows that $\rho_{z_{i}}^{ \pm} \circ \alpha_{i}=\rho_{\tilde{z}_{i}}^{ \pm} \circ \iota$ for $i=1,2$. Thus, $\tilde{z}_{1} \stackrel{\tau, M_{ \pm}^{1}}{\sim} z_{1}$ and $\tilde{z}_{2} \stackrel{\tau, M_{ \pm}^{2}}{\sim} z_{2}$. By Lemma 3.13 (i) and (ii), $s=\Phi\left[z_{1}, z_{2}\right]\left(\alpha_{1}, \alpha_{2}\right)=\Phi\left[\tilde{z}_{1}, \tilde{z}_{2}\right](\iota, \iota) \in \mathscr{I}_{2}\left[z_{1}, z_{2}\right]$.

Now, suppose $s \in \mathscr{I}_{2}$, that is, $s=\Phi\left[\tilde{z}_{1}, \tilde{z}_{2}\right](\iota, \iota)$ for $\tilde{z}_{1} \stackrel{\tau, M_{ \pm}^{1}}{\sim} z_{1}$ and $\tilde{z}_{2} \stackrel{\tau, M_{ \pm}^{2}}{\sim} z_{2}$. Hence, there exist $\alpha_{i}, \beta_{i} \in \mathscr{A}_{\tau}$ such that $\rho_{z_{i}}^{ \pm} \circ \alpha_{i}=\rho_{\tilde{z}_{i}}^{ \pm} \circ \beta_{i}$ for $i=1,2$. Since $0 \leq M_{ \pm}^{i}<\infty$ we might as well assume that $\beta_{i}(0)<\beta_{i}(t)$ for any $0<t \leq \tau$. Hence, by Lemma 3.16 , there exist sequences $\left\{\zeta_{i}^{\varepsilon}\right\}_{\varepsilon>0} \subset \mathscr{A}_{\tau}$ such that $\beta_{i} \circ \zeta_{i}^{\varepsilon} \downarrow \iota$ uniformly as $\varepsilon \downarrow 0$ for $i=1,2$. By Lemma 3.13 (i) and (iii),

$$
\begin{aligned}
s^{\varepsilon, \eta}: & =\Phi\left[\tilde{z}_{1}, \tilde{z}_{2}\right]\left(\beta_{1} \circ \zeta_{1}^{\varepsilon}, \beta_{2} \circ \zeta_{2}^{\eta}\right) \\
& =\Phi\left[z_{1}, z_{2}\right]\left(\alpha_{1} \circ \zeta_{1}^{\varepsilon}, \alpha_{2} \circ \zeta_{2}^{\eta}\right) \in \mathscr{I}_{1}\left[z_{1}, z_{2}\right] .
\end{aligned}
$$

By Lemma 3.13 (ii), there exist subsequences $\left\{\varepsilon_{j}\right\}_{j \geq 0},\left\{\eta_{j}\right\}_{j \geq 0}$ such that $\varepsilon_{j}, \eta_{j} \downarrow 0$ and $s^{\varepsilon_{j}, \eta_{j}} \rightarrow s$, as $j \rightarrow \infty$.

\section{REFERENCES}

[1] L. Ambrosio, N. Fusco, and D. Pallara. Functions of bounded variation and free discontinuity problems. Oxford Mathematical Monographs, The Clarendon Press, Oxford University Press, New York, 2000. MR 18572923.1

[2] E. Audusse, S. Boyaval, Y. Gao, and D. Hilhorst. Numerical simulations of the inviscid Burgers equation with periodic boundary conditions and stochastic forcing. In CEMRACS 2013modelling and simulation of complex systems: stochastic and deterministic approaches, volume 48 of ESAIM Proc. Surveys, pages 308-320. EDP Sci., Les Ulis, 2015. 1

[3] S. Attanasio and F. Flandoli. Renormalized solutions for stochastic transport equations and the regularization by bilinear multiplication noise. Comm. Partial Differential Equations, 36(8):1455-1474, 2011. 1

[4] I. Bailleul and M. Gubinelli. Unbounded rough drivers. ArXiv e-prints, Jan. 2015. 1

[5] C. Bauzet. Time-splitting approximation of the cauchy problem for a stochastic conservation law. Mathematics and Computers in Simulation, 118:73-86, 2015. 1

[6] C. Bauzet, J. Charrier, and T. Gallouët. Convergence of flux-splitting finite volume schemes for hyperbolic scalar conservation laws with a multiplicative stochastic perturbation. Math. Comp., 85(302):2777-2813, 2016. 1

[7] C. Bauzet, J. Charrier, and T. Gallouët. Convergence of monotone finite volume schemes for hyperbolic scalar conservation laws with multiplicative noise. Stoch. Partial Differ. Equ. Anal. Comput., 4(1):150-223, 2016. 1

[8] C. Bauzet, G. Vallet, and P. Wittbold. The Cauchy problem for conservation laws with a multiplicative stochastic perturbation. J. Hyperbolic Differ. Equ., 9(4):661-709, 2012. 1

[9] I. H. Biswas, K. H. Karlsen, and A. K. Majee. Conservation laws driven by Lévy white noise. J. Hyperbolic Differ. Equ., 12(3):581-654, 2015. 1

[10] G.-Q. Chen and K. H. Karlsen. Quasilinear anisotropic degenerate parabolic equations with time-space dependent diffusion coefficients. Commun. Pure Appl. Anal., 4(2):241-266, 2005.

[11] G.-Q. Chen, Q. Ding, and K. H. Karlsen. On nonlinear stochastic balance laws. Arch. Ration. Mech. Anal., 204(3):707-743, 2012. 1 
[12] C. M. Dafermos. Hyperbolic conservation laws in continuum physics, volume 325 of Grundlehren der Mathematischen Wissenschaften [Fundamental Principles of Mathematical Sciences]. Springer-Verlag, Berlin, third edition, 2010. 1, $1,3.2,3.2,3.2$

[13] A. Debussche and J. Vovelle. Scalar conservation laws with stochastic forcing. J. Funct. Anal., 259(4):1014-1042, 2010. 1

[14] A. Debussche and J. Vovelle. Invariant measure of scalar first-order conservation laws with stochastic forcing. Probab. Theory Related Fields, 163(3-4):575-611, 2015. 1

[15] A. Debussche, M. Hofmanová, and J. Vovelle. Degenerate parabolic stochastic partial differential equations: Quasilinear case. Ann. Probab., 44(3):1916-1955, 2016. 1

[16] A. Deya, M. Gubinelli, M. Hofmanová, and S. Tindel. A priori estimates for rough PDEs with application to rough conservation laws. ArXiv e-prints, Apr. 2016. 1

[17] S. Dotti and J. Vovelle. Convergence of approximations to stochastic scalar conservation laws. ArXiv e-prints, Nov. 2016. 1

[18] S. Dotti and J. Vovelle. Convergence of the Finite Volume Method for scalar conservation laws with multiplicative noise: an approach by kinetic formulation. ArXiv e-prints, Nov. 2016. 1

[19] W. E, K. Khanin, A. Mazel, and Y. Sinai. Invariant measures for Burgers equation with stochastic forcing. Ann. of Math. (2), 151(3):877-960, 2000. 1

[20] J. Feng and D. Nualart. Stochastic scalar conservation laws. J. Funct. Anal., 255(2):313-373, 2008. 1

[21] F. Flandoli, M. Gubinelli, and E. Priola. Well-posedness of the transport equation by stochastic perturbation. Invent. Math., 180(1):1-53, 2010. 1

[22] P. Gassiat and B. Gess. Regularization by noise for stochastic Hamilton-Jacobi equations. ArXiv e-prints, Sept. 2016. 2.7 2.7 1

[23] B. Gess, B. Perthame, and P. E. Souganidis. Semi-discretization for stochastic scalar conservation laws with multiple rough fluxes. SIAM J. Numer. Anal., 54(4):2187-2209, 2016.

[24] B. Gess and P. E. Souganidis. Long-time behavior, invariant measures and regularizing effects for stochastic scalar conservation laws. ArXiv e-prints, Nov. 2014. 1

[25] B. Gess and P. E. Souganidis. Scalar conservation laws with multiple rough fluxes. Commun. Math. Sci., 13(6):1569-1597, 2015. 1

[26] H. Hoel, K. H. Karlsen, N. H. Risebro, and E. B. Storrøsten. Numerical methods for stochastic conservation laws with rough flux Preprint, 2017. (document) 1

[27] M. Hofmanová. Degenerate parabolic stochastic partial differential equations. Stochastic Process. Appl., 123(12):4294-4336, 2013. 1

[28] M. Hofmanová. Scalar conservation laws with rough flux and stochastic forcing. Stoch. Partial Differ. Equ. Anal. Comput., 4(3):635-690, 2016. 1

[29] H. Holden and N. H. Risebro. Conservation laws with a random source. Appl. Math. Optim., 36(2):229-241, 1997. 1 .

[30] K. H. Karlsen and E. B. Storrøsten. On stochastic conservation laws and Malliavin calculus. J. Funct. Anal., 272:421-497, 2017. 1

[31] K. H. Karlsen and E. B. Storrøsten. Analysis of a splitting method for stochastic balance laws. IMA J. Numer. Anal., to appear. 1

[32] J. U. Kim. On a stochastic scalar conservation law. Indiana Univ. Math. J. 52 (1) (2003) 227-256. 1

[33] I. Kröker and C. Rohde. Finite volume schemes for hyperbolic balance laws with multiplicative noise. Appl. Numer. Math., 62(4):441-456, 2012. 1

[34] P.-L. Lions, B. Perthame, and P. Souganidis. Scalar conservation laws with rough (stochastic) fluxes. Stoch. Partial Differ. Equ. Anal. Comput., 1(4):664-686, 2013. (document) 1] 1

[35] P.-L. Lions, B. Perthame, and P. E. Souganidis. Stochastic averaging lemmas for kinetic equations. ArXiv e-prints, Apr. 2012. 1

[36] P.-L. Lions, B. Perthame, and P. E. Souganidis. Scalar conservation laws with rough (stochastic) fluxes: the spatially dependent case. Stoch. Partial Differ. Equ. Anal. Comput., 2(4):517-538, 2014. 1

[37] S.-E. A. Mohammed, T. K. Nilssen, and F. N. Proske. Sobolev differentiable stochastic flows for SDEs with singular coefficients: Applications to the transport equation. Ann. Probab., 43(3):1535-1576, 2015. 1

[38] W. Neves and C. Olivera. Wellposedness for stochastic continuity equations with Ladyzhenskaya-Prodi-Serrin condition. NoDEA Nonlinear Differential Equations Appl., 22(5):1247-1258, 2015. 1 
[39] B. Perthame. Kinetic formulation of conservation laws, volume 21 of Oxford Lecture Series in Mathematics and its Applications. Oxford University Press, Oxford, 2002. 1

[40] G. Vallet. Dirichlet problem for a nonlinear conservation law. Rev. Mat. Complut., 13(1):231250, 2000. 1

[41] G. Vallet and P. Wittbold. On a stochastic first-order hyperbolic equation in a bounded domain. Infin. Dimens. Anal. Quantum Probab. Relat. Top., 12(4):613-651, 2009. 1

[42] C. Villani. Topics in optimal transportation, volume 58 of Graduate Studies in Mathematics. American Mathematical Society, Providence, RI, 2003.2.9

(Håkon Hoel)

Mathematics Institute of Computational Science and Engineering

ÉCole Polytechnique fÉdÉrale de LAUSANNe

EPFL / SB / MATH-CSQI, MA C1 644 (BÂtiment MA), Station 8

CH-1015, Lausanne

SWITZERLAND

(Kenneth Hvistendahl Karlsen)

DEPARTMENT OF MATHEMATICS

UNIVERSITY OF OSLO

P.O. Box 1053, BLindern

N-0316 Oslo, Norway

E-mail address:k.h.karlsen@me.com

(Nils Henrik Risebro)

Department of mathematics

UNIVERSITY OF OSLO

P.O. Box 1053, Blindern

N-0316 OsLo, Norway

E-mail address: nilshr@math.uio.no

(Erlend Briseid Storrøsten)

Department of MATHEMATICS

UNIVERSITY OF OSLO

P.O. Box 1053, BLINDERN

N-0316 OsLo, Norway

E-mail address: erlenbs@math.uio.no 